\title{
The asymptotic behavior of bootstrap support values in molecular phylogenetics
}

\author{
Jun HuAng ${ }^{1,2}$, Yuting LiU ${ }^{1, *}$, Tiandi ZhU ${ }^{3, *}$ AND ZiHeng YANG ${ }^{2, *}$ \\ ${ }^{1}$ Department of Mathematics, Beijing Jiaotong University, Beijing, 100044, China \\ ${ }^{2}$ Department of Genetics, Evolution and Environment, University College London, Gower Street, London WC1E \\ 6BT, UK. \\ ${ }^{3}$ Academy of Mathematics and Systems Science, Chinese Academy of Sciences, Beijing 100190, China. \\ * Correspondence to be sent to: Yuting Liu, Department of Mathematics, Beijing Jiaotong University, Beijing, \\ 100044, China; E-mail: ytliu@bjtu.edu.cn \\ Tianqi Zhu, Academy of Mathematics and Systems Science, Chinese Academy of Sciences, Beijing 100190, China; \\ E-mail: zhutq@amss.ac.cn \\ Ziheng Yang, Department of Genetics, Evolution and Environment, University College London, Gower Street, \\ London WC1E 6BT, UK; E-mail: z.yang@ucl.ac.uk (orcid: 0000-0003-3351-7981)
}

\begin{abstract}
The phylogenetic bootstrap is the most commonly used method for assessing statistical confidence in estimated phylogenies by non-Bayesian methods such as maximum parsimony and maximum likelihood (ML). It is observed that bootstrap support tends to be high in large genomic datasets whether or not the inferred trees and clades are correct. Here we study the asymptotic behavior of bootstrap support for the ML tree in large datasets when the competing phylogenetic trees are equally right or equally wrong. We consider phylogenetic reconstruction as a problem of statistical model selection when the compared models are nonnested and misspecified. The bootstrap is found to have qualitatively different dynamics from Bayesian inference, and does not exhibit the polarized behavior of posterior model probabilities, consistent with the empirical observation that the bootstrap is more conservative than Bayesian probabilities. Nevertheless bootstrap support similarly shows fluctuations among large datasets, with no convergence to a point value, when the compared models are equally right or equally wrong. Thus in large datasets strong support for wrong trees or models is likely to occur. Our analysis provides a partial explanation for the high bootstrap support values for incorrect clades observed in empirical data analysis.
\end{abstract}

Key words: Bootstrap, model selection, star-tree paradox, support value

\section{INTRODUCTION}

Recently Yang and Zhu (2018) characterized the asymptotic behaviors of Bayesian model selection in large datasets. When two models are both right or are equally wrong and indistinct, the posterior model probability varies among datasets according to a statistical distribution such as $\mathbb{U}(0,1)$, whereas one might expect it to converge to the point value $\frac{1}{2}$. Even more disturbingly, when the two models are equally wrong and distinct, the posterior model probability approaches $\sim 100 \%$ in some datasets and $0 \%$ in others. This polarized behavior may be a major reason for the observation that in Bayesian analysis of large phylogenetic datasets, posterior probabilities for trees or clades are most often close to $100 \%$, whether or not the relationships are correct. 
For non-Bayesian methods including maximum parsimony (Fitch, 1971), neighbor joining (Saitou and Nei, 1987), and maximum likelihood (ML, Felsenstein, 1981), confidence for inferred trees or clades is most often assessed using Felsenstein's (1985) phylogenetic bootstrap. An interesting question is whether bootstrap exhibits similar behaviors as the posterior model probabilities. In modern phylogenomic studies both posterior probabilities and bootstrap support values tend to be $\sim 100 \%$, whether or not the clades or trees are correct. Such results lead to widespread mistrust for bootstrap support values in large datasets. For example, Chan et al. (2020) wrote that "high bootstrap support did not necessarily reflect congruence or support for the correct topology. This study reiterates findings of some previous studies, which demonstrated that traditional bootstrap values can produce positively misleading measures of support in large phylogenomic datasets."

Bootstrap was originally developed by Efron (1979) to calculate the standard error for a parameter, by resampling the original data and studying the variation among the bootstrap resample datasets. It has since been used to conduct all sorts of analyses in Frequentist statistics, such as correction for bias, calculation of standard errors, construction of confidence intervals, and performing significance tests (Efron and Tibshirani, 1993; Davison and Hinkley, 1997). In phylogenetics, bootstrap was introduced by Felsenstein (1985) to assess the confidence in estimated phylogenetic trees. Although it follows the same operational procedure of resampling data points from the observed dataset, bootstrap in phylogenetics differs from its use in bias correction or in confidence-interval construction, in that a statistical interpretation has been illusory despite numerous efforts (Zharkikh and Li, 1992; Hillis and Bull, 1993; Felsenstein and Kishino, 1993; Berry and Gascuel, 1996; Efron et al., 1996; Holmes, 2003; Susko, 2009). Modifications to the procedure have also been made, including the complete-and-partial bootstrap (Zharkikh and Li, 1995), correction for first-order biases (Susko, 2010), or adjustment for short branches (Lemoine et al., 2018). These correct for the perceived bias in the procedure or to make it agree better with standard ideas of confidence levels and hypothesis testing.

Its interpretation aside, phylogenetic bootstrap is the most widely used procedure for assessing the confidence in estimated phylogenies by non-Bayesian methods. Felsenstein's 1985 paper is a citation classic in all sciences. For Bayesian methods, the posterior probability for the inferred tree provides a natural measure of uncertainty (Rannala and Yang, 1996), and bootstrap is in theory not needed in Bayesian inference. However, the sensitivity of Bayesian model choice to the prior (O'Hagan and Forster, 2004) and the polarized behavior of Bayesian model selection under model misspecification (Yang and Zhu, 2018) have prompted the application of bootstrap in Bayesian model selection as well, leading to methods such as Bayesian bagging (Rubin, 1981; Weng, 1989; Huggins and Miller, 2020). It is important to study the asymptotic behavior of phylogenetic bootstrap. Earlier simulation studies suggest that the phylogenetic bootstrap may be conservative, and that 70\% (instead of 95\%) means strong support (e.g., Hillis and Bull, 1993). It has been noted that bootstrap support is numerically less extreme than posterior model probabilities (e.g., Huelsenbeck and Rannala, 2004; Yang and Rannala, 2005).

In this paper we explore the asymptotic behavior of phylogenetic bootstrap when the data size increases. We consider phylogenetic reconstruction as a statistical model selection problem, and treat phylogenetic trees as nonnested statistical models (rather than different values of a parameter in a well-specified model). We present an asymptotic theory for bootstrap model probability under different scenarios in the Appendix, and in the main paper illustrate the theory using canonical problems that are analytically tractable. We discuss phylogenetic reconstruction problems in the case of three or four taxa to illustrate the general theory.

\section{SUMMARY OF ANALYTICAL RESULTS}

Following Felsenstein and Kishino (1993) and Efron et al. (1996), we consider bootstrap as a general approach to assessing the confidence in the selected model in a model-selection problem. 
Bootstrap in model selection

The data are an independently and identically distributed (i.i.d.) sample of size $n, x=$ $\left\{x_{1}, \cdots, x_{n}\right\}$, from the true data-generating model $g(X)$. We compare $K$ models, $H_{j}, j=1, \cdots, K$. Model $H_{j}$ specifies the density $f_{j}\left(X \mid \theta_{j}\right)$ with parameters $\theta_{j}$. Let $\hat{\theta}_{j}$ be the MLE of $\theta_{j}$ under model $H_{j}$ given data $x$. When $n \rightarrow \infty, \hat{\theta}_{j} \rightarrow \theta_{j *}$, where $\theta_{j *}$ minimizes the Kullback-Leibler (K-L) divergence from model $H_{j}$ to the true model,

$$
D_{j}=\int g(X) \log \frac{g(X)}{f_{j}\left(X \mid \theta_{j^{*}}\right)} \mathrm{d} X \text {. }
$$

If $H_{j}$ is correct, $\theta_{j *}$ will be the true parameter values, with $D_{j}=0$. Otherwise if $H_{j}$ is wrong, $\theta_{j *}$ will be the best-fitting or pseudo-true parameter values, with $D_{j}>0$. In this paper we focus on the case where all $K$ models have the same K-L divergence to the true model. Two models $f_{1}$ and $f_{2}$ are said to be equally right if $D_{1}=D_{2}=0$, and equally wrong if $D_{1}=D_{2}>0$. If two models are unidentifiable at their pseudo-true parameter values, that is, if

$$
f_{1}\left(X \mid \theta_{1 *}\right)=f_{2}\left(X \mid \theta_{2 *}\right) \text { for almost every } X,
$$

they are said to be indistinct. This can occur when both models are right (with $D_{1}=D_{2}=0$ ) or when both are wrong (with $D_{1}=D_{2}>0$ ). Otherwise if equation (2) does not hold for some $X$ of nonzero measure, the models are said to be distinct. This can occur only if both models are wrong (with $D_{1}=D_{2}>0$ ).

The model selected by ML is the one that achieves the greatest log likelihood, $\ell_{j}\left(\hat{\theta}_{j}\right)=$ $\log f_{j}\left(x \mid \hat{\theta}_{j}\right)$. To assess the confidence on the selected model, we calculate the bootstrap probability. Let $x_{b}^{*}=\left\{x_{b 1}^{*}, \ldots, x_{b n}^{*}\right\}$ be a bootstrap sample, formed by resampling with replacement $n$ times from the original data $x$. Let $\hat{\theta}_{b}^{*}$ be the MLE from a bootstrap sample $x^{*}$. Here we follow the convention of using the superscript * to indicate a bootstrap sample, and the subscript ${ }_{*}$ for the true or pseudo-true parameter values. We assume that $\theta_{j *}, \hat{\theta}_{j}$, and $\hat{\theta}_{j}^{*}$ are inner points in the parameter space. The proportion of bootstrap replicates in which model $j$ is the optimal model is the bootstrap probability or bootstrap support $P_{j}$ for model $j$. For example, in the case of two models, the bootstrap probability for model $H_{1}$ is

$$
P_{1}(x)=\mathbb{P}\left\{\log f_{1}\left(x^{*} \mid \hat{\theta}_{1}^{*}\right)>\log f_{2}\left(x^{*} \mid \hat{\theta}_{2}^{*}\right) \mid x\right\} \approx \frac{1}{B} \sum_{b} \mathbb{I}_{\ell_{1}\left(\hat{\theta}_{1}^{*}\right)>\ell_{2}\left(\hat{\theta}_{2}^{*}\right)},
$$

where $\ell_{j}\left(\hat{\theta}_{j}^{*}\right)=\log f_{j}\left(x^{*} \mid \hat{\theta}_{j}^{*}\right)$ is the log likelihood value for model $j$, calculated at the $\operatorname{MLE}\left(\hat{\theta}_{j}^{*}\right)$ and where the indicator function $\mathbb{I}_{A}$ is 1 if $A$ is true or 0 otherwise. Note that $P_{1}$ is a function of $x$ and is a random variable. We are interested in the asymptotic distribution of $P_{1}$ when $x$ varies.

In phylogenetics, the models under comparison are the tree topologies for the given set of species, while each data point corresponds to one site or one column in the alignment. While the bootstrap is applicable as long as the inference method is statistically consistent (Felsenstein, 1985), we focus on ML in this paper. In phylogenetics, bootstrap is commonly used to attach support values for clades or splits on the phylogeny, calculated as the proportion of bootstrap trees that contain the splits. Here we focus on the bootstrap probability for the whole model. In the case of simple trees with three or four species with only one internal branch, the two measures are equivalent. We assume that the number of bootstrap replicates $B$ is large so that the sampling errors due to limited number of bootstrap replicates is negligible. 
.

The asymptotic behavior of bootstrap model selection under different scenarios

We develop an asymptotic theory of bootstrap model selection in the Appendix. In general, when equally right or equally wrong models are compared, bootstrap model probabilities have a non-degenerate distribution. In the case of two equally wrong and distinct models, the bootstrap model probability $P_{1}$ has the distribution $\mathbb{U}(0,1)$.

The case of two equally wrong and distinct models with no parameters provides valuable insights into the differences between bootstrap and Bayesian methods. The log-likelihood ratio between the two models is

$$
\Delta=\log \frac{f_{1}(x)}{f_{2}(x)}, \quad \Delta^{*}=\log \frac{f_{1}\left(x^{*}\right)}{f_{2}\left(x^{*}\right)},
$$

for the original data $x$ and the bootstrap resample data $x^{*}$, respectively. Each of these is a sum of $n$ i.i.d. terms. Thus $\mathbb{E}(\Delta)=n \mathbb{E} \log f_{1}(X)-n \mathbb{E} \log f_{2}(X)=n\left(D_{2}-D_{1}\right)=0$ (Equation 1). Let

$$
\sigma^{2}=\mathbb{V}\left\{\log \frac{f_{1}(X)}{f_{2}(X)}\right\}=\int g(X)\left[\log \frac{f_{1}(X)}{f_{2}(X)}\right]^{2} \mathrm{~d} X .
$$

When $n \rightarrow \infty, \Delta \sim \mathbb{N}\left(0, n \sigma^{2}\right)$ and $\Delta^{*} \mid x \sim \mathbb{N}\left(\Delta, n \sigma^{2}\right)$, according to the central limit theorem. Thus

$$
P_{1}=\mathbb{P}\left\{\Delta^{*}>0 \mid x\right\}=\Phi\left(\frac{\Delta}{\sqrt{n} \sigma}\right) \rightarrow \mathbb{U}(0,1),
$$

where $\Phi$ is the cumulative distribution function (CDF) for $\mathbb{N}(0,1)$.

In Bayesian comparison of two equally wrong models with no parameters, $\Delta$ is the log Bayes factor. With equal prior probabilities ( $\frac{1}{2}$ for each model), this is related to the posterior model probability through $\Delta=\log \frac{P_{1}}{1-P_{1}}$ or $P_{1}=\frac{\mathrm{e}^{\Delta}}{\mathrm{e}^{\Delta}+1}$. As $\Delta$ behaves like a random walk when $n$ increases, it is nearly impossible for $\Delta$ to be in a small interval around 0 , say, $-5<\Delta<5$ which corresponds to $0.007<P_{1}<0.993$. In other words, for large $n$, the posterior probability will be 0 in half of the datasets and 1 in the other half. This polarized behavior also occurs when the compared models, equally wrong and distinct, have parameters as the Bayes factor is dominated by the random-walk term (Yang and Zhu, 2018). The analysis here suggests that bootstrap probability has a qualitatively different behavior, as it contrasts $\Delta^{*}$ for the bootstrap sample with $\Delta$ for the original data.

We present several simple examples to illustrate the asymptotic behavior of bootstrap model probability under different scenarios when the data size $n \rightarrow \infty$. In the first two examples two models are equally wrong and distinct, and the bootstrap probability $P_{1}$ varies among datasets like a random number, $P_{1} \sim \mathbb{U}(0,1)$ (Equation 6).

Problem 1 fair-coin paradox, with equally wrong models and no parameters. Suppose a coin is fair with the true probability of heads to be $p=0.5$, and we flip the coin $n$ times to compare two models $H_{1}: p=0.4$ and $H_{2}: p=0.6$. The dataset is $x=\left\{x_{1}, \ldots, x_{n}\right\}$, where $x_{i}$ takes the value 1 for heads and 0 for tails, and has the bernoulli distribution. The data can be summarized as the proportion of heads in $n$ tosses, $\bar{x}$, which is approximately normal $\mathbb{N}\left(\frac{1}{2}, \frac{1}{4 n}\right) . H_{1}$ is favored if $\bar{x}<\frac{1}{2}$, and this happens in half of the datasets.

Given $x$, the bootstrap sample $x_{b}^{*}=\left\{x_{b 1}^{*}, \cdots, x_{b n}^{*}\right\}$, where $x_{b i}^{*}$ is bernoulli with probability $\bar{x}$, can be summarized as the bootstrap sample mean $\bar{x}^{*}$, which is approximately normal, with $\bar{x}^{*} \mid x \sim \mathbb{N}\left(\bar{x}, \frac{\bar{x}(1-\bar{x})}{n}\right) \approx \mathbb{N}\left(\bar{x}, \frac{1}{4 n}\right)$. The bootstrap sample $x_{b}^{*}$ favors model $H_{1}$ if and only if $\bar{x}^{*}<1 / 2$. Thus 
(A) Problem 1

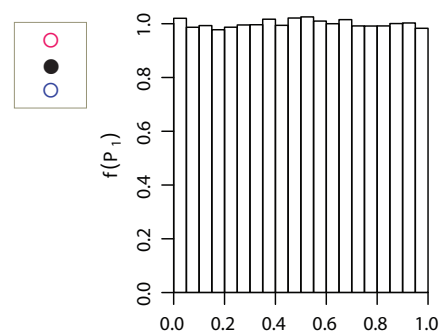

(C) Problem 3
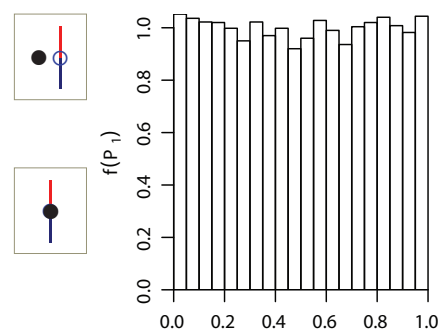

(B) Problem 2

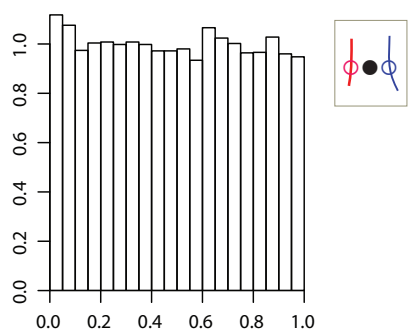

(D) Problem 4

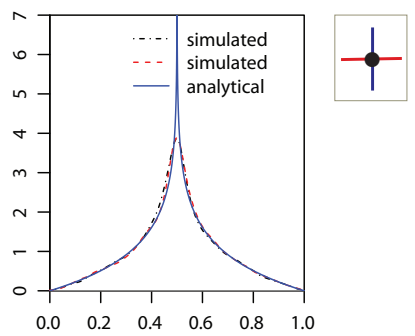

Fig. 1. Histogram/density of bootstrap model probability $P_{1}$ in comparisons of two models. (A) Problem 1 (the fair-coin paradox) in which a fair coin (with $p=0.5$ ) is tossed $n$ times to compare two equally wrong and distinct models: $p=0.4$ and $p=0.6$. (B) Problem 2 in which the true model is $\mathbb{N}(0,1)$ while the two compared models, $\mathbb{N}\left(\mu, 1 / \tau_{1}\right)$ and $\mathbb{N}\left(\mu, 1 / \tau_{2}\right)$ with $\tau_{1}<1<\tau_{2}$, are equally wrong and distinct. (C) Problem 3 (the fair-balance paradox) where the true model is $\mathbb{N}(0,1)$ and the two compared models, $\mathbb{N}(\mu, 1 / \tau), \mu<0$ versus $\mathbb{N}(\mu, 1 / \tau), \mu>0$, are equally right (if $\tau=1$ ) or equally wrong and indistinct (if $\tau \neq 1$ ). (D) Problem 4 (equally right models). The true model is $\mathbb{N}(0,1)$ while the two compared models, $\mathbb{N}(\mu, 1)$ versus $\mathbb{N}(0,1 / \tau)$, are both right. Black dashed line is for the expensive simulation generating $x$ and $x^{*}$, the red dashed line is for simulation generating $\bar{x}$ and $s^{2}$, while blue solid line is for the analytical approximation by Equation 14. The insets characterize the problems, with the true models represented as filled circles and the pseudo-true parameter values as empty circles, while the lines represent the parameter space for each model. The settings are $n=10^{5}, B=3 \times 10^{4}$, and $R=10^{5}$ for problem $1, n=10^{4}, B=3 \times 10^{4}$, and $R=10^{4}$ for problem $2, n=10^{4}, B=3 \times 10^{4}$, and $R=10^{4}$ for problem 3 , and $n=10^{4}, B=10^{3}$, and $R=10^{4}$ for problem 4 .

$$
P_{1}=\mathbb{P}\left\{\bar{x}^{*}<\frac{1}{2} \mid x\right\} \approx \Phi\left(\frac{1 / 2-\bar{x}}{\sqrt{1 /(4 n)}}\right) \rightarrow \mathbb{U}(0,1), \quad \text { as } n \rightarrow \infty .
$$

Thus $P_{1}$ varies like a random number among datasets (Fig. 1A). Alternatively we have $\Delta=$ $\ell_{1}-\ell_{2}=2 n\left(\bar{x}-\frac{1}{2}\right) \log \frac{0.4}{0.6} \sim \mathbb{N}\left(0, n \sigma^{2}\right)$ and $\Delta^{*} \mid x \sim \mathbb{N}\left(\Delta, n \sigma^{2}\right)$, with $\sigma=\log \frac{0.4}{0.6}$, so that Equation 6 gives $P_{1} \sim \mathbb{N}(0,1)$.

Problem 2 Normal distribution, equally wrong and distinct models with free parameters. Suppose the true model is $\mathbb{N}(0,1)$ and we consider $H_{1}: \mathbb{N}\left(\mu, 1 / \tau_{1}\right)$ and $H_{2}: \mathbb{N}\left(\mu, 1 / \tau_{2}\right)$, where $\mu$ is a free parameter while the precisions $\tau_{1}$ and $\tau_{2}$ are given with $\log \left(\tau_{2} / \tau_{1}\right)=\tau_{2}-\tau_{1}$ so that the two models are equally wrong $\left(D_{1}=D_{2}>0\right)$ (Yang and Zhu, 2018). We use $\tau_{1}=0.25$ and $\tau_{2}=2.58666$. Under each model, the pseudo-true parameter value is $\mu_{*}=0$ and $H_{1}$ and $H_{2}$ are two equally wrong and distinct models. Note that $H_{1}$ is over-dispersed and $H_{2}$ is under-dispersed. Under the model $\mathbb{N}(\mu, 1 / \tau)$ with known $\tau$, the log likelihood is

$$
\ell=-\frac{n}{2} \log (2 \pi)+\frac{n}{2} \log \tau-\frac{\tau}{2} \sum_{i=1}^{n}\left(x_{i}-\mu\right)^{2},
$$

with $\hat{\mu}=\bar{x}$. Thus $\ell_{1}>\ell_{2}$ if and only if $\left(\tau_{2}-\tau_{1}\right) \sum_{i=1}^{n}\left(x_{i}-\bar{x}\right)^{2}>n \log \left(\tau_{2} / \tau_{1}\right)$ or if and only if $s^{2}=\frac{1}{n} \sum_{i=1}^{n}\left(x_{i}-\bar{x}\right)^{2}>1$. We have $n s^{2} \sim \chi_{n-1}^{2} \approx \mathbb{N}(n-1,2(n-1))$. 
Given $x$, the bootstrap sample $x^{*}$ favors $H_{1}$ if the sample variance $s^{2 *}>1$. We have $n s^{2 *} / s^{2} \mid x \sim \chi_{n-1}^{2} \approx \mathbb{N}(n-1,2(n-1))$. For large $n$, re-sampling from the empirical distribution represented by the observed data $x$ is approximately equivalent to sampling from the continuous distribution $\mathbb{N}\left(\bar{x}, s^{2}\right)$. Thus

$$
P_{1}=\mathbb{P}\left\{s^{2 *}>1 \mid x\right\} \approx \Phi\left(\frac{((n-1) / n) s^{2}-1}{\sqrt{2(n-1) / n^{2}}}\right) \rightarrow \mathbb{U}(0,1), \quad \text { as } n \rightarrow \infty .
$$

This is confirmed in Fig. 1B.

Alternatively we have $\Delta=\ell_{1}-\ell_{2}=\frac{n}{2}\left(\tau_{2}-\tau_{1}\right)\left(s^{2}-1\right) \sim \mathbb{N}\left(0, n \sigma^{2}\right)$ and $\Delta^{*} \mid \Delta \sim$ $\mathbb{N}\left(\Delta, n \sigma^{2}\right)$, with $\sigma=\frac{1}{\sqrt{2}}\left(\tau_{2}-\tau_{1}\right)$, so that $P_{1}=\mathbb{P}\left\{\Delta^{*}>0 \mid x\right\} \sim \mathbb{N}(0,1)$.

If the two compared models are both right (with $D_{1}=D_{2}=0$ ) or are equally wrong and indistinct (with $D_{1}=D_{2}>0$ ), then $P_{1}$ varies among datasets according to a nondegenerate distribution, which may and may not be $\mathbb{U}(0,1)$ ), as illustrated in the next two examples.

Problem 3 (fair-balance paradox with two equally right or equally wrong and indistinct models). The true model is $\mathbb{N}(0,1)$ and the two compared models are $\mathbb{N}(\mu, 1 / \tau)$, $\mu<0$ and $\mathbb{N}(\mu, 1 / \tau), \mu>0$, with $\tau$ given. If $\tau=1$, the two models are equally right. If $\tau \neq 1$, the two models are equally wrong (because of the assumed incorrect variance) and indistinct (because the pseudo-true parameter value $\mu_{*}=0$ under each model). Model 1 is favored if and only if the sample mean $\bar{x}<0$. As $\bar{x} \sim \mathbb{N}(0,1 / n)$ and $\bar{x}^{*} \mid x \sim \mathbb{N}(\bar{x}, 1 / n)$, we have

$$
P_{1}=\mathbb{P}\left\{\bar{x}^{*}<0 \mid x\right\}=\Phi(-\sqrt{n} \bar{x}) \rightarrow \mathbb{U}(0,1), \quad \text { as } n \rightarrow \infty .
$$

This is confirmed in Fig. 1C.

Problem 4 Normal-distribution example with an infinite spike at $\frac{1}{2}$ in the $P_{1}$

distribution. The true model is $\mathbb{N}(0,1)$ and the two compared models are $\mathbb{N}(\mu, 1)$ and $\mathbb{N}(0,1 / \tau)$. In $H_{1}, \mu_{*}=0$ while in $H_{2}, \tau_{*}=1$, so the two models are equally right. The data $x$ may be summarized as the sample mean $\bar{x}$ and sample variance $s^{2}=\frac{1}{n} \sum_{i}\left(x_{i}-\bar{x}\right)^{2}$. The MLE of the parameter is $\hat{\mu}=\bar{x}$ under $H_{1}$ and $\hat{\tau}=n / \sum x_{i}^{2}=1 /\left(s^{2}+\bar{x}^{2}\right)$ under $H_{2}$. The log-likelihood values are

$$
\begin{aligned}
& \ell_{1}(\hat{\mu})=-\frac{1}{2} \sum\left(x_{i}-\bar{x}\right)^{2}=-\frac{1}{2} n s^{2}, \\
& \ell_{2}(\hat{\tau})=-\frac{n}{2} \log \left(\frac{1}{n} \sum x_{i}^{2}\right)-\frac{n}{2}=-\frac{n}{2} \log \left(s^{2}+\bar{x}^{2}\right)-\frac{n}{2}
\end{aligned}
$$

Thus $\ell_{1}>\ell_{2}$ if and only if

$$
\bar{x}^{2}>\mathrm{e}^{s^{2}-1}-s^{2} \approx 1+\left(s^{2}-1\right)+\frac{1}{2}\left(s^{2}-1\right)^{2}-s^{2}=\frac{1}{2}\left(s^{2}-1\right)^{2},
$$

or if and only if

$$
|\bar{x}|>\frac{1}{\sqrt{2}}\left|s^{2}-1\right| .
$$

A large deviation of $\bar{x}$ from 0 supports $H_{1}$, whereas a large deviation of $s^{2}$ from 1 favors $H_{2}$. Also $\bar{x} \sim \mathbb{N}\left(0, \frac{1}{n}\right)$ and $s^{2} \sim \frac{1}{n} \chi_{n-1}^{2} \approx \mathbb{N}\left(\frac{n-1}{n}, \frac{2(n-1)}{n^{2}}\right)$ or $\frac{1}{\sqrt{2}}\left(s^{2}-1\right) \sim \mathbb{N}\left(0, \frac{1}{n}\right)$, and $\bar{x}$ and $s^{2}$ are independent. Thus Equation 13 holds and $H_{1}$ is the selected model in half of the datasets.

Given $x$, we have $\bar{x}^{*} \mid x \sim \mathbb{N}\left(\bar{x}, s^{2} / n\right) \approx \mathbb{N}\left(\bar{x}, \frac{1}{n}\right)$ and $\frac{1}{\sqrt{2}}\left(s^{2 *}-1\right) \mid x \sim \mathbb{N}\left(\frac{1}{\sqrt{2}}\left(s^{2}-1\right), \frac{1}{n}\right)$ and $\bar{x}^{*}$ and $s^{2 *}$ are conditionally independent. Let $z_{1}=\sqrt{n} \bar{x}$ and $z_{2}=\sqrt{\frac{n}{2}}\left(s^{2}-1\right)$, with $z_{1}$ and $z_{2}$ from $\mathbb{N}(0,1)$. Let $z_{1}^{*}=\sqrt{n} \bar{x}^{*}$ and $z_{2}^{*}=\sqrt{\frac{n}{2}}\left(s^{2 *}-1\right)$, with $z_{1}^{*} \mid x \sim \mathbb{N}\left(z_{1}, 1\right)$ and $z_{2}^{*} \mid x \sim \mathbb{N}\left(z_{2}, 1\right)$ to be conditionally i.i.d. Then

$$
P_{1}=\mathbb{P}\left\{\left|\bar{z}_{1}^{*}\right|>\left|\bar{z}_{2}^{*}\right| \mid x\right\} .
$$


This problem is analyzed in the SI text, available on Dryad at https://doi.org/10.5061/dryad.7m0cfxprw. The limiting distribution of $P_{1}$ when $n \rightarrow \infty$ is

$$
f\left(P_{1}\right)=-\log \left|2 P_{1}-1\right| .
$$

The density is symmetrical around $\frac{1}{2}$, is 0 at 0 and 1 , and has an infinite spike at $\frac{1}{2}$, with the mean $\frac{1}{2}$ and variance $\frac{1}{36}$. This is confirmed by simulation in Fig. 1D. The simulation is done in two ways. In the first, data $x$ is sampled from $\mathbb{N}(0,1)$, and given $x$ bootstrap samples $x_{b}^{*}$ are generated, with $\bar{x}^{*}$ and $s^{2 *}$ calculated to apply Equation 14 . In the second approach, $\bar{x} \sim \mathbb{N}(0,1 / n)$ and $n s^{2} \sim \chi_{n-1}^{2}$ are sampled, and then $\bar{x}^{*} \sim \mathbb{N}\left(\bar{x}, s^{2} / n\right)$ and $n s^{2 *} / s^{2} \sim \chi_{n-1}^{2}$ are generated to select the model for the bootstrap sample using Equation 14. Both approaches produce the same results as Equation 15.

Problem 5 multivariate normal-distribution example. The true model is the $(K-1)$-variate normal distribution $\mathbb{N}(\mu, \Sigma)$, with mean vector $\mu=\left(\mu_{1}, \cdots, \mu_{K-1}\right)$ where $\mu_{1}=$ $\cdots=\mu_{K-1}=0$ and variance matrix $\Sigma$ which has 1 on the diagonal and $-1 /(K-1)$ on the off-diagonal. The data are an i.i.d. sample of size $n, x=\left\{x_{i j}\right\}, i=1, \cdots, n ; j=1, \cdots, K-1$. Also let $x_{i K}=-\left(x_{i 1}+\cdots+x_{i, K-1}\right)$ and $\mu_{K}=-\left(\mu_{1}+\cdots+\mu_{K-1}\right)$. We use the data to compare $K$ models. Model $H_{j}, j=1, \cdots, K$, assumes $\mu_{j}>\mu_{k}$ for any $k \neq j$. The model has $K-1$ free parameters: $\mu_{1}, \cdots, \mu_{K}$ with the constraint $\mu_{1}+\cdots+\mu_{K}=0$. The variance is assumed to be known, $c \Sigma$. The models are equally right if $c=1$ and equally wrong if $c \neq 1$. An alternative formulation of the problem is to have only one parameter in model $H_{j}: \mu_{j}>\mu_{k}$ with $\mu_{k}=-\mu_{j} /(K-1)$ for all $k \neq j$

Let $\bar{x}=\left\{\bar{x}_{j}\right\}$ and $\bar{x}^{*}=\left\{\bar{x}_{j}^{*}\right\}$, with

$$
\bar{x}_{j}=\frac{1}{n} \sum_{i} x_{i j}, \quad \bar{x}_{j}^{*}=\frac{1}{n} \sum_{i} x_{i j}^{*}, \quad j=1, \cdots, K,
$$

be the sample means from dataset $x$ and from bootstrap sample $x^{*}$, respectively. Then $\bar{x} \sim \mathbb{N}\left(\mu, \frac{1}{n} \Sigma\right)$ and approximately $\bar{x}^{*} \mid x \sim \mathbb{N}\left(\bar{x}, \frac{1}{n} \Sigma\right)$. Without the constraint under each model $H_{j}$ : $\mu_{j}>\mu_{k}$, the MLEs of $\mu$ are the sample means. With the constraint, $H_{j}$ is the selected model if $\bar{x}_{j}$ is the greatest among $\bar{x}_{1}, \cdots, \bar{x}_{K}$. The bootstrap probability for model $H_{1}$ given data $x$ is

$$
P_{1}=\mathbb{P}\left(\bar{x}_{1}^{*}>\bar{x}_{2}^{*}, \cdots, \bar{x}_{1}^{*}>\bar{x}_{K}^{*} \mid x\right) .
$$

Now for any $j \neq k$,

$$
\sigma_{j k}^{2}=\mathbb{V}\left(\bar{x}_{j}-\bar{x}_{k}\right)=\frac{2}{n}-2 \cdot \frac{1}{n} \cdot\left(-\frac{1}{K-1}\right)=\frac{2}{n} \cdot \frac{K}{K-1} .
$$

Let $z=\left(z_{2}, \cdots, z_{K}\right)^{T}$ and $z^{*}=\left(z_{2}^{*}, \cdots, z_{K}^{*}\right)^{T}$, with $z_{j}=\frac{\bar{x}_{1}-\bar{x}_{j}}{\sigma_{1 j}}$ and $z_{j}^{*}=\frac{\bar{x}_{1}^{*}-\bar{x}_{j}^{*}}{\sigma_{1 j}}, j=2, \cdots K$. We have

$$
\begin{aligned}
& \mathbb{V}\left(z_{j}\right)=1, \\
& \begin{aligned}
\operatorname{Cor}\left(z_{j}, z_{k}\right) & =\operatorname{Cov}\left(\bar{x}_{1}-\bar{x}_{j}, \bar{x}_{1}-\bar{x}_{k}\right) /\left(\sigma_{1 j} \sigma_{1 k}\right) \\
& =\left[\mathbb{V}\left(\bar{x}_{1}\right)-2 \operatorname{Cov}\left(\bar{x}_{1}, \bar{x}_{j}\right)+\operatorname{Cov}\left(\bar{x}_{i}, \bar{x}_{j}\right)\right] /\left(\sigma_{1 j} \sigma_{1 k}\right) \\
& =\frac{1}{n}\left(1+\frac{1}{K-1}\right) /\left(\frac{2}{n} \frac{K}{K-1}\right)=\frac{1}{2} .
\end{aligned}
\end{aligned}
$$

Thus $z \sim \mathbb{N}\left(0, \Sigma_{0}\right)$ and $z^{*} \mid x \sim \mathbb{N}\left(z, \Sigma_{0}\right)$, where $\Sigma_{0}$ is a $(K-1) \times(K-1)$ variance matrix with 1 on the diagonal and $\frac{1}{2}$ on the off-diagonal. Thus

$$
P_{1}=\mathbb{P}\left(z_{2}^{*}>0, \cdots, z_{K}^{*}>0 \mid x\right)=\Phi\left(z_{2}, \cdots, z_{K}\right) .
$$




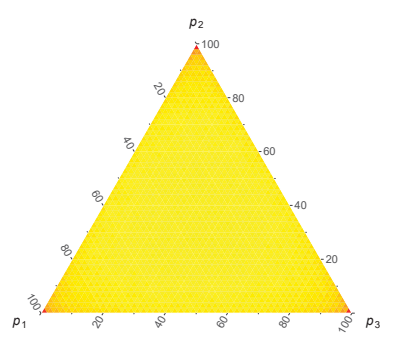

(A)

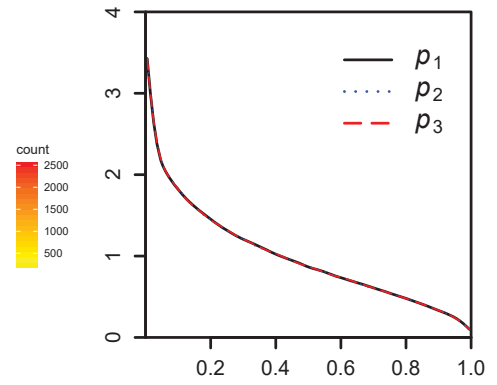

(B)

Fig. 2. Marginal and joint distributions of $P_{1}, P_{2}, P_{3}$ for problem 5 (the multivariate normal example with $K=3$ ). The three corners in the plots correspond to points $(1,0,0),(0,1,0)$, and $(0,0,1)$, while the center is $\left(\frac{1}{3}, \frac{1}{3}, \frac{1}{3}\right)$. The number of replicates is $R=10^{6}$, with $n=10^{6}$ and $B=10^{3}$.
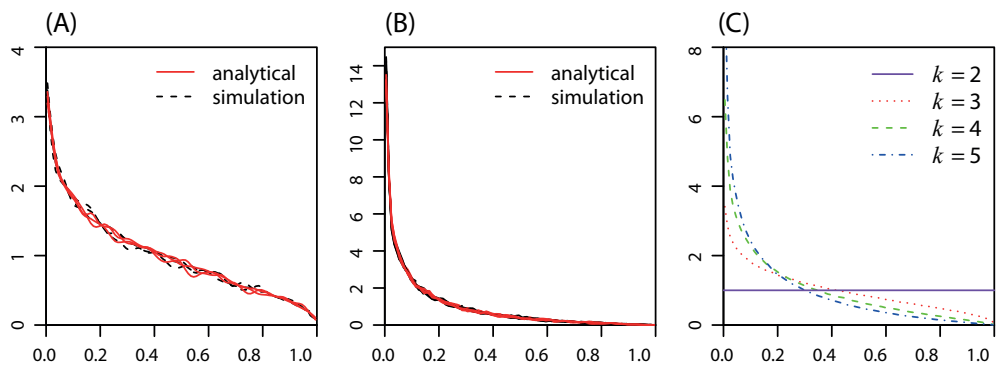

Fig. 3. Marginal distribution of $P_{1}$ in comparisons of $K$ equally right or equally wrong and indistinct models based on the normal distribution of Problem 5 ( $K=3$ in A and 6 in B). The sample size is $n=10^{4}$. The number of simulated replicates is $R=10^{4}$, with $B=10^{3}$, but the 'theoretical' distribution is based on simulating $10^{6}$ replicates and using Equation 21 .

As $\bar{x}_{j}^{*}-\bar{x}_{k}^{*}=\left(\bar{x}_{1}^{*}-\bar{x}_{k}^{*}\right)-\left(\bar{x}_{1}^{*}-\bar{x}_{j}^{*}\right)$, the bootstrap probabilities for all $K$ models given data $x$ are

$$
\left[\begin{array}{c}
P_{1} \\
P_{2} \\
\vdots \\
P_{K}
\end{array}\right]=\left[\begin{array}{c}
\Phi\left(z_{2}, z_{3}, \cdots, z_{K}\right) \\
\Phi\left(-z_{2}, z_{3}-z_{2}, \cdots, z_{K}-z_{2}\right) \\
\vdots \\
\Phi\left(-z_{K}, z_{2}-z_{K}, \cdots, z_{K-1}-z_{K}\right)
\end{array}\right]
$$

For example, in the case of $K=3$, a fast way of simulating the limiting distribution of $\left(P_{1}, P_{2}, P_{3}\right)$ is thus to generate $\left(z_{2}, z_{3}\right) \sim \mathbb{N}\left(\left(\begin{array}{l}0 \\ 0\end{array}\right),\left(\begin{array}{cc}1 & 0.5 \\ 0.5 & 1\end{array}\right)\right)$ and then calculate $\left(P_{1}, P_{2}, P_{3}\right)$ by Equation 21. This is confirmed by the slow simulation of generating $x$ and then $x^{*}$ in Fig. 2. The joint distribution of $\left(P_{1}, P_{2}, P_{3}\right)$ has peaks at the three corners, and is nearly flat around the center. By symmetry $P_{1}$ has mean $\frac{1}{3}$, and by numerical integration using Equation 20, $P_{1}$ has $\mathrm{SD}=$ 0.25904 . The probability that one of the models is strongly supported is close to 0 (table 1). Fig. 3A\&B shows the marginal distribution of $P_{1}$ when $K=3$ and 6 .

Even though $\left(P_{1}, P_{2}, P_{3}\right)$ do not converge to the point value $\left(\frac{1}{3}, \frac{1}{3}, \frac{1}{3}\right)$, extreme bootstrap support values are not highly frequent. Bootstrap probabilities are thus qualitatively different from Bayesian model probabilities. 
Table 1. Proportions of data replicates with very high bootstrap probability $\left(P_{1}\right)$ in the multivariate normal example (Problem 5)

\begin{tabular}{ccccc}
\hline$K$ & 2 & 3 & 4 & 5 \\
\hline $\mathbb{P}\left\{P_{1}>0.90\right\}$ & 0.100 & 0.023 & 0.008 & 0.004 \\
$\mathbb{P}\left\{P_{1}>0.95\right\}$ & 0.050 & 0.008 & 0.003 & 0.001 \\
$\mathbb{P}\left\{P_{1}>0.99\right\}$ & 0.010 & 0.001 & 0.000 & 0.000 \\
\hline
\end{tabular}

A
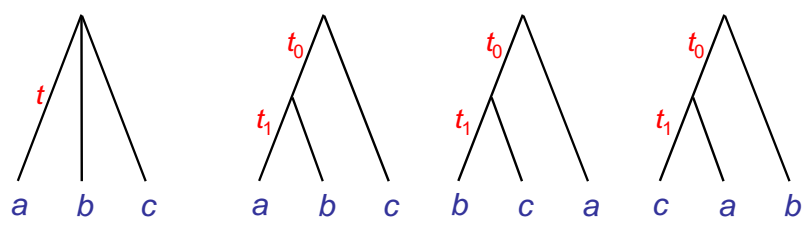

$T_{0}$

$T_{1}$

$T_{2}$

$T_{3}$
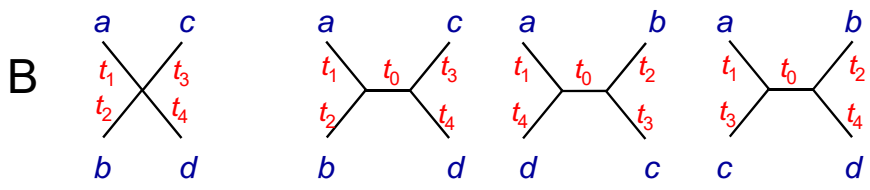

Fig. 4. The star tree $T_{0}$ and three binary rooted trees $T_{1}, T_{2}$, and $T_{3}$ for (A) three or (B) four species. Branch length parameters, shown next to the branches, are measured by the expected number of changes per site. The star tree is used to generate data, which are analyzed by ML to compare the three binary trees.

\section{BOOTSTRAP IN PHYLOGENETICS}

We consider ML reconstruction of phylogenies of three or four species (Fig. 4), under the JC model (Jukes and Cantor, 1969). We simulate data to verify the asymptotic theory and compare with Bayesian results from Yang and Zhu (2018).

Case A (Fig. 5A\&A') involves equally right models. This is the star-tree paradox analyzed previously (Lewis et al., 2005; Yang and Rannala, 2005; Yang, 2007a; Susko, 2008). We use the rooted star tree $T_{0}$ for three species with $t=0.2$ (Fig. 4A) to generate datasets to compare the three binary trees. The JC model (Jukes and Cantor, 1969) is used both to generate and to analyze the data. The molecular clock (rate constancy over time) is assumed as well, so that the parameters in each binary tree are the two node ages $\left(t_{0}\right.$ and $\left.t_{1}\right)$, measured by the expected number of nucleotide changes per site. The best-fitting parameter values are $t_{0 *}=0$ and $t_{1 *}=0.2$ for each of the three binary trees, so that the three binary trees are equally right models.

Case B (Fig. 5B\&B') involves equally wrong models that are indistinct. This is similar to case A except that the JC $+\Gamma$ model (Jukes and Cantor, 1969; Yang, 1993) is used to generate data, with different sites in the sequence evolving at variable rates according to the gamma distribution with shape parameter $\alpha=1$. The data are then analyzed using JC (equivalently to $\mathrm{JC}+\Gamma$ with $\alpha=\infty$ ), giving $t_{0 *}=0$ and $t_{1 *}=0.16441$ as the pseudo-true parameter values for each binary tree. The binary trees are equally wrong and indistinct models $\left(D_{1}=D_{2}=D_{3}>0\right)$.

Case C (Fig. 5C\&C') involves equally wrong and distinct models. Like case B, the simulation model is $\mathrm{JC}+\Gamma$ with $\alpha=1$ and the analysis model is $\mathrm{JC}$. However, the molecular clock is not assumed and unrooted trees are used. The true tree is the unrooted star tree $T_{0}$ of Fig. 4B, with $t_{1}=t_{2}=t_{3}=t_{4}=0.2$, with $t_{0 *}=0.01037$ and $t_{i *}=0.16409, i=1, \cdots, 4$ for the binary trees (Fig. 4B). As $t_{0 *}>0$, the three binary trees are equally wrong and distinct models $\left(D_{1}=D_{2}=\right.$ $\left.D_{3}>0\right)$.

In cases $\mathrm{A}$ and $\mathrm{B}$, the data for three species have a multinomial distribution with five 


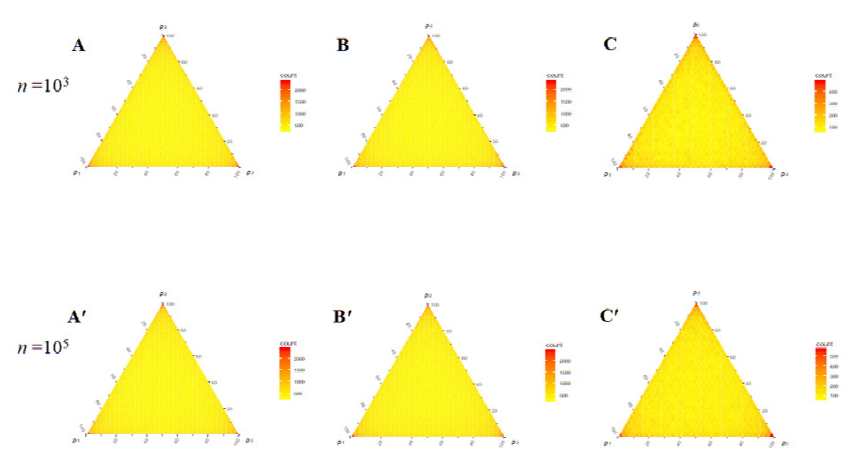

Fig. 5. The joint distribution of the bootstrap model probabilities in the star-tree problem. The star tree $T_{0}$ of Fig. 4 is used to simulate data (sequence alignments of $n=10^{3}$ or $10^{5}$ sites), and $\mathrm{ML}$ is used to compare the three binary trees $T_{1}, T_{2}$, and $T_{3}$ to calculate their bootstrap probabilities $\left(P_{1}, P_{2}, P_{3}\right)$. In (A) and (A'), the true tree is the star tree $T_{0}$ for three species of Fig. 4A, with $t=0.2$. Both the simulation and analysis models are JC, and the three binary trees are equally right models. In (B) and (C), the true tree is the star tree $T_{0}$ for three species of Fig. $4 \mathrm{~A}$, with $t=0.2$. The simulation model is $\mathrm{JC}+\Gamma$ (with $\alpha=1$ ), and the analysis model is JC. The three binary trees represent equally wrong and indistinct models. In (C) and (C'), the true tree is the star tree $T_{0}$ for four species of Fig. 4B, with $t_{1}=t_{2}=t_{3}=t_{4}=0.2$. The simulation model is $\mathrm{JC}+\Gamma(\alpha=1)$ and the analysis model is JC. The three binary trees represent equally wrong and distinct models. The number of bootstrap samples $B=1000$ and the number of replicates is $R=10^{6}$ for three-species trees and $10^{4}$ for four-species trees.

categories corresponding to the five site patterns $x x x, x x y, x y x, y x x$, and $x y z$, where $x, y, z$ are any distinct nucleotides. Let the frequencies of the informative site patterns $x x y, x y x, y x x$ be $\bar{x}_{1}, \bar{x}_{2}$, and $\bar{x}_{3}$, while that for the two uninformative patterns $x x x$ and $x y z$ be $\bar{x}_{0}$. With the star tree being the true tree, the probabilities for the three informative site patterns are identical, with $p_{1}=p_{2}=p_{3}$. Tree 1 specifies $p_{1}>p_{2}=p_{3}$. Given data $x$, tree $j$ is the ML tree if $\bar{x}_{j}$ is the greatest among $\bar{x}_{1}, \bar{x}_{2}$, and $\bar{x}_{3}$ (Yang, 2000). Then $\bar{x}=\left(\bar{x}_{1}, \bar{x}_{2}, \bar{x}_{3}\right)$ is approximately normal, with mean $(p, p, p)$, and variance $p(1-p) / n$ and covariance $-p^{2} / n$. Applying a multivariate normal approximation to the multinomial distribution, we see that the problem has the same mathematical structure as problem 5. Thus the bootstrap distribution for cases A and B should be identical to that in problem 5. We wrote a $\mathrm{C}$ program to simulate and analyze data for cases A and B. Given branch lengths $t_{0}$ and $t_{1}$, the probabilities for the five site patterns are calculated according to the JC model (Yang, 1994), and the data $x$ are then generated by sampling from the multinomial distribution. Given data $x$, bootstrap dataset $x^{*}$ is sampled using the observed site-pattern frequencies in $x$. Then tree $j$ is the ML tree for data $x^{*}$ if $\bar{x}_{j}^{*}$ is the largest among $\left(\bar{x}_{1}^{*}, \bar{x}_{2}^{*}, \bar{x}_{3}^{*}\right)$.

In case $\mathrm{C}$ for four species, the informative site patterns are $x x y y, x y x y$ and $x y y x$ while there are 11 uninformative patterns. The binary tree has only five parameters, such that the model achieves a better fit to the observed data by having a positive internal branch length. As a result, the three binary trees are distinct models (with $t_{0 *}>0$ ). Case $\mathrm{C}$ thus differs from problem 5 , but has a similar symmetry in that the K-L distance between any pair of models is the same. From the general theory, the distribution of bootstrap probabilities $\left(P_{1}, P_{2}, P_{3}\right)$ is the same as that in problem 5 . We simulated data using EVOLVER, and generated bootstrap resample data using SEQBOOT. The data are then analyzed using BASEML in PAML (Yang, 2007b).

Our theory predicts that the limiting distribution is the same in all three cases, with the mean $1 / 3$ and SD 0.25904 . This is confirmed by the simulation (Fig. 5), which gave the mean of $P_{1}$ as $1 / 3$ and the SD as 0.259 . The bootstrap probabilities have modes at the corners, and roughly uniformly distributed around the center. While in case $\mathrm{C}$, the Bayesian posterior probabilities show extreme polarized behavior, concentrated on three points: $(1,0,0),(0,1,0)$, and $(0,0,1)$ (Yang and Zhu, 2018, Fig. 4C\&4C'), bootstrap probabilities are much more moderate and have a nondegenerate distribution. 
Table 2. Proportions of datasets with extreme bootstrap or posterior (in parentheses) probabilities for the three binary trees in the star-tree simulation

\begin{tabular}{ccccccc}
$n$ & $\mathbb{P}\left\{P_{\min }<1 \%\right\}$ & $\mathbb{P}\left\{P_{\min }<5 \%\right\}$ & $\mathbb{P}\left\{P_{\max }>95 \%\right\}$ & $\mathbb{P}\left\{P_{\max }>99 \%\right\}$ & $\mathbb{E}\left(P_{\min }\right)$ & $\mathbb{E}\left(P_{\max }\right)$ \\
\hline $10^{3}$ & $0.119(0.234)$ & $0.391(0.550)$ & $0.028(0.205)$ & $0.001(0.079)$ & $0.094(0.067)$ & $0.644(0.754)$ \\
$10^{4}$ & $0.123(0.812)$ & $0.386(0.931)$ & $0.030(0.606)$ & $0.002(0.450)$ & $0.093(0.011)$ & $0.653(0.897)$ \\
$10^{5}$ & $0.113(0.979)$ & $0.383(0.992)$ & $0.029(0.853)$ & $0.004(0.773)$ & $0.093(0.001)$ & $0.647(0.964)$ \\
\hline
\end{tabular}

Note. $-P_{\min }=\min \left(P_{1}, P_{2}, P_{3}\right)$ and $P_{\max }=\max \left(P_{1}, P_{2}, P_{3}\right)$. Data are generated under $\mathrm{JC}+\Gamma$ with $\alpha$ $=1$, using the star tree for four species $(a: 0.2, b: 0.2, c: 0.2, d: 0.2)$, and analyzed under JC. The number of replicates is $R=10^{3}$ and the number of bootstrap samples is $B=10^{3}$. The probability density of $\left(P_{1}, P_{2}, P_{3}\right)$ is shown in Fig. 5C\&C' for $n=10^{3}$ and $10^{5}$, respectively. Posterior tree probabilities from the Bayesian analysis are shown in parentheses, from Yang and Zhu (2018, table S1).

Table 3. Proportions of datasets with strong bootstrap or posterior (in parentheses) support for wrong trees in simulated datasets for four species

\begin{tabular}{ccccc}
\hline$n$ & $\mathbb{P}\left\{P_{1}<1 \%\right\}$ & $\mathbb{P}\left\{P_{1}<5 \%\right\}$ & $\mathbb{P}\left\{P_{23}>95 \%\right\}$ & $\mathbb{P}\left\{P_{23}>99 \%\right\}$ \\
\hline $10^{3}$ & $0.031(0.083)$ & $0.109(0.225)$ & $0.019(0.113)$ & $0.002(0.038)$ \\
$10^{4}$ & $0.009(0.250)$ & $0.044(0.337)$ & $0.005(0.266)$ & $0.000(0.166)$ \\
$10^{5}$ & $0.000(0.102)$ & $0.001(0.120)$ & $0.000(0.115)$ & $0.000(0.097)$ \\
\hline
\end{tabular}

Note. $-P_{1}$ is the probability for the true tree, while $P_{2}$ and $P_{3}$ are for the two wrong trees, with $P_{23}=\max \left\{P_{2}, P_{3}\right\}$. Data were generated under $\mathrm{JC}+\Gamma$ with $\alpha=1$ on the unrooted tree $T_{1}$ for four species: $((a: 0.2, b: 0.2): 0.002, c: 0.2, d: 0.2)$, and analyzed under JC. The number of simulated replicates is $R=10^{3}$, with $B=10^{3}$. Posterior tree probabilities from the Bayesian analysis are shown in parentheses, from Yang and Zhu (2018, table S2).

We also calculated the proportions of datasets in which the bootstrap and posterior probabilities for the three binary trees are extremely high (table 2 ). When the sequence length is $n=10^{5}, \mathbb{E}\left(P_{\max }\right)=0.647$ using bootstrap method and 0.964 for the Bayesian method. If $P_{\max }>0.95$, one of the models is strongly favored, and this occurs in $2.9 \%$ of datasets for the bootstrap and $85.3 \%$ for the Bayesian. In other words, it is much less likely to see high bootstrap support for equally wrong models than high posterior probabilities for them.

DISCUSSION

As mentioned in Introduction, the interpretation of bootstrap in model selection in general and in phylogenetics in particular is controversial. A number of studies have attempted to give bootstrap a Bayesian interpretation, that is, the bootstrap probability for a tree is the probability that the tree is correct. For example, Hastie et al. (2009, p.272) wrote that "[i]n this sense, the bootstrap distribution represents an (approximate) nonparametric, noninformative posterior distribution for our parameter." The plug-in principle for bootstrap appears to support this interpretation: bootstrap probability $\mathbb{P}\left\{\Delta^{*}>0 \mid x\right\}$ is an estimate of $\mathbb{P}\{\Delta>0\}$, which is the probability that the ML tree is correct. In phylogenetics, such an interpretation was suggested by Efron et al. (1996), although the assumed prior for the corresponding Bayesian analysis has infinite branch lengths and is implausible biologically (Yang, 2014, p.176).

Our analysis suggests qualitatively different asymptotic behaviors between bootstrap and 
posterior probabilities for models or trees. The greatest difference occurs in the case of comparing equally wrong and distinct models. In that case, the posterior model probabilities show extreme polarized behavior, with $\sim 100 \%$ for one model and 0 for others. This behavior is because the log marginal likelihood ratio for two models (or the log Bayes factor) $(\Delta)$ is dominated by a random-walk that deviates from 0 (which corresponds to the posterior probability $\frac{1}{2}$ for each model) at the rate of $\sqrt{n}$ when $n$ increases Yang and Zhu (2018), so that for large $n$, the posterior model probability is either 0 or 1 . Bootstrap probabilities show a different behavior. While the $\log$ likelihood ratio for the bootstrap dataset $\left(\Delta^{*}\right)$ also increases like a random walk when $n$ increases, it is compared with the log likelihood ratio for the original dataset $(\Delta)$ when the bootstrap model probability is calculated. As a result, whether the models are distinct or indistinct does not matter anymore.

\section{ACKNOWLEDGMENTS}

We thank Professor Zhi-ming Ma for discussions. This study has been supported by Biotechnology and Biological Sciences Research Council grant (BB/P006493/1) to Z.Y. and a BBSRC equipment grant (BB/R01356X/1). J.H.'s visit to UCL is supported by China Scholarship Council (CSC).

D

Data available from the Dryad Digital Repository: https://doi.org/10.5061/dryad.7m0cfxprw.

\section{SUPPLEMENTAL DATA}

REFERENCES

Berry, V. and Gascuel, O. 1996. On the interpretation of bootstrap trees: appropriate threshold of clade selection and induced gain. Mol. Biol. Evol., 13: 999-1011.

Bickel, P. J. and Freedman, D. A. 1981. Some asymptotic theory for the bootstrap. Ann. Statist., 9: 1196-1217.

Chan, K. O., Hutter, C. R., Wood, P. L., J., Grismer, L. L., and Brown, R. M. 2020. Larger, unfiltered datasets are more effective at resolving phylogenetic conflict: Introns, exons, and uces resolve ambiguities in golden-backed frogs (anura: Ranidae; genus hylarana). Mol. Phylogenet. Evol., 151: 106899.

Cheng, G. and Huang, J. Z. 2010. Bootstrap consistency for general semiparametric M-estimation. Ann. Statist., 38(5): 2884-2915.

DasGupta, A. 2008. The bootstrap. In Asymptotic Theory of Statistics and Probability, pages 461-497. Springer, New York.

Davison, A. and Hinkley, D. 1997. Bootstrap Methods and their Application. Cambridge University Press, Cambridge, UK.

Dawid, A. 2011. Posterior model probabilities. In P. S. Bandyopadhyay and M. Forster, editors, Philosophy of Statistics, pages 607-630. Elsevier, New York.

Efron, B. 1979. Bootstrap methods: another look at the jackknife. Ann. Stat., 7: 1-26.

Efron, B. and Tibshirani, R. 1993. An Introduction to the Bootstrap. Chapman and Hall, London.

Efron, B., Halloran, E., and Holmes, S. 1996. Bootstrap confidence levels for phylogenetic trees. Proc. Natl. Acad. Sci. U.S.A., 93: 13429-13434. 
Felsenstein, J. 1981. Evolutionary trees from DNA sequences: a maximum likelihood approach. J. Mol. Evol., 17(6): 368-376.

Felsenstein, J. 1985. Confidence limits on phylogenies: an approach using the bootstrap. Evolution, 39: 783-791.

Felsenstein, J. and Kishino, H. 1993. Is there something wrong with the bootstrap on phylogenies? a reply to Hillis and Bull. Syst. Biol., 42(2): 193-200.

Fitch, W. M. 1971. Toward defining the course of evolution: minimum change for a specific tree topology. Syst. Zool., 20: 406-416.

Hastie, T., Tibshirani, R., and Friedman, J. 2009. The Elements of Statistical Learning: Data Mining, Inference, and Prediction. Springer, New York, 2 edition.

Hillis, D. M. and Bull, J. J. 1993. An empirical test of bootstrapping as a method for assessing confidence in phylogenetic analysis. Syst. Biol., 42: 182-192.

Holmes, S. 2003. Bootstrapping phylogenetic trees: theory and methods. Stat. Sci., 18: 241-255.

Huelsenbeck, J. and Rannala, B. 2004. Frequentist properties of bayesian posterior probabilities of phylogenetic trees under simple and complex substitution models. Syst. Biol., 53: 904-913.

Huggins, J. H. and Miller, J. W. 2020. Using bagged posteriors for robust inference and model criticism. page arXiv: 1912.07104 .

Jukes, T. and Cantor, C. 1969. Evolution of protein molecules. In H. Munro, editor, Mammalian Protein Metabolism, pages 21-123. Academic Press, New York.

Lemoine, F., Domelevo Entfellner, J.-B., Wilkinson, E., Correia, D., Davila Felipe, M., De Oliveira, T., and Gascuel, O. 2018. Renewing Felsenstein's phylogenetic bootstrap in the era of big data. Nature, 556: 452-456.

Lewis, P., Holder, M., and Holsinger, K. 2005. Polytomies and bayesian phylogenetic inference. Syst. Biol., 54: 241-253.

O'Hagan, A. and Forster, J. 2004. Kendall's Advanced Theory of Statistics: Bayesian Inference. Arnold, London.

Rannala, B. and Yang, Z. 1996. Probability distribution of molecular evolutionary trees: a new method of phylogenetic inference. J. Mol. Evol., 43: 304-311.

Rubin, D. B. 1981. The Bayesian bootstrap. Ann. Statist., 9(1): 130-134.

Saitou, N. and Nei, M. 1987. The neighbor-joining method: a new method for reconstructing phylogenetic trees. Mol. Biol. Evol., 4: 406-425.

Susko, E. 2008. On the distributions of bootstrap support and posterior distributions for a star tree. Syst. Biol., 57: 602-612.

Susko, E. 2009. Bootstrap support is not first-order correct. Syst. Biol., 58(2): 211-223.

Susko, E. 2010. First-order correct bootstrap support adjustments for splits that allow hypothesis testing when using maximum likelihood estimation. Mol. Biol. Evol., 27: 1621-1629.

Weng, C.-S. 1989. On a second-order asymptotic property of the bayesian bootstrap mean. Ann. Statist., 17(2): 705-710. 
White, H. 1982. Maximum likelihood estimation of misspecified models. Econometrica, 50: $1-25$.

Yang, Z. 1993. Maximum-likelihood estimation of phylogeny from DNA sequences when substitution rates differ over sites. Mol. Biol. Evol., 10: 1396-1401.

Yang, Z. 1994. Statistical properties of the maximum likelihood method of phylogenetic estimation and comparison with distance matrix methods. Syst. Biol., 43: 329-342.

Yang, Z. 2000. Complexity of the simplest phylogenetic estimation problem. Proc. R. Soc. B: Biol. Sci., 267: 109-116.

Yang, Z. 2007a. Fair-balance paradox, star-tree paradox and bayesian phylogenetics. Mol. Biol. Evol., 24: 1639-1655.

Yang, Z. 2007b. PAML 4: Phylogenetic analysis by maximum likelihood. Mol. Biol. Evol., 24: $1586-1591$.

Yang, Z. 2014. Molecular Evolution: A Statistical Approach. Oxford University Press, Oxford, England.

Yang, Z. and Rannala, B. 2005. Branch-length prior influences bayesian posterior probability of phylogeny. Syst. Biol., 54: 455-470.

Yang, Z. and Zhu, T. 2018. Bayesian selection of misspecified models is overconfident and may cause spurious posterior probabilities for phylogenetic trees. Proc. Natl. Acad. Sci. USA, 115(8): 1854-1859.

Zharkikh, A. and Li, W.-H. 1992. Statistical properties of bootstrap estimation of phylogenetic variability from nucleotide sequences. i. four taxa with a molecular clock. Mol. Biol. Evol., 9: $1119-1147$.

Zharkikh, A. and Li, W.-H. 1995. Estimation of confidence in phylogeny: the complete-and-partial bootstrap technique. Mol. Phylogenet. Evol., 4: 44-63. 


\section{APPENDIX. ASYMPTOTIC THEORY FOR BOOTSTRAP PROBABILITY IN MODEL SELECTION}

We use ML to compare $K$ models, $H_{j}: X \sim f_{j}\left(X \mid \theta_{j}\right), j=1, \cdots, K$. The dataset, $x=\left\{x_{1}, \cdots, x_{n}\right\}$, is an i.i.d. sample of from the true model $g(X)$. Given $x$, we generate a bootstrap sample $x^{*}$ and analyze it using ML. The bootstrap probability for model $H_{1}$ is the probability that model $H_{1}$ has higher log likelihood than other models in the bootstrap sample.

The case of two equally wrong and distinct models. We decompose the log-likelihood ratio between models $H_{1}$ and $H_{2}$ for the bootstrap dataset $x^{*}$ into several components, and study their dynamics when $n \rightarrow \infty$.

$$
\Delta^{*} \equiv \log \frac{f_{1}\left(x^{*} \mid \hat{\boldsymbol{\theta}}_{1}^{*}\right)}{f_{2}\left(x^{*} \mid \hat{\boldsymbol{\theta}}_{2}^{*}\right)}=\log \frac{f_{1}\left(x^{*} \mid \hat{\boldsymbol{\theta}}_{1}^{*}\right)}{f_{1}\left(x^{*} \mid \hat{\boldsymbol{\theta}}_{1}\right)}-\log \frac{f_{2}\left(x^{*} \mid \hat{\boldsymbol{\theta}}_{2}^{*}\right)}{f_{2}\left(x^{*} \mid \hat{\boldsymbol{\theta}}_{2}\right)}+\log \frac{f_{1}\left(x^{*} \mid \hat{\theta}_{1}\right)}{f_{2}\left(x^{*} \mid \hat{\theta}_{2}\right)} \equiv \Delta A_{1}-\Delta A_{2}+\Delta_{*}^{*} .
$$

Model $H_{1}$ is the selected model in the bootstrap sample if and only if $\Delta^{*}>0$, so that the bootstrap probability for $H_{1}$ given data $x$ is $P_{1} \equiv \mathbb{P}\left\{\Delta^{*}>0 \mid x\right\}$. We are interested in the distribution of $P_{1}$ when $x$ varies. First we consider the case where $H_{1}$ and $H_{2}$ are equally wrong and distinct. We show that $\Delta A_{1}$ and $\Delta A_{2}$ are $O_{p}(1)$ while $\Delta_{*}^{*}$ is $O_{p}\left(n^{1 / 2}\right)$, so that $\Delta^{*}$ is dominated by $\Delta_{*}^{*}$.

Taking the same approach as in Dawid (2011) and Yang and Zhu (2018), we apply Taylor expansion to the $\log$ likelihood, $\log f_{1}\left(x^{*} \mid \theta_{1}\right)$, for the bootstrap dataset $x^{*}$ around the MLE $\hat{\theta}_{1}^{*}$ and then let $\theta_{1}=\hat{\theta}_{1}$. We have

$$
\begin{aligned}
\Delta A_{1} & =\log f_{1}\left(x^{*} \mid \hat{\theta}_{1}^{*}\right)-\log f_{1}\left(x^{*} \mid \hat{\theta}_{1}\right) \\
& \approx \frac{1}{2}\left\{\left(\hat{\theta}_{1}^{*}-\hat{\theta}_{1}\right)\right\}^{T}\left(n J_{1}\left(\hat{\theta}_{1}^{*}\right)\right)\left\{\left(\hat{\theta}_{1}^{*}-\hat{\theta}_{1}\right)\right\} \\
& \approx \frac{1}{2}\left\{\sqrt{n}\left(\hat{\theta}_{1}-\theta_{1 *}\right)\right\}^{T} J_{1}\left(\theta_{1 *}\right)\left\{\sqrt{n}\left(\hat{\theta}_{1}-\theta_{1 *}\right)\right\},
\end{aligned}
$$

where $J_{1}\left(\theta_{1}\right)=\mathbb{E}\left\{-\nabla^{2} \log f_{1}\left(X \mid \theta_{1}\right)\right\}$ and $\nabla^{2}$ is the second derivatives with respect to $\theta_{1}$. From the plug-in principle, $x^{*}$ varies given $\hat{\theta}$ as does $x$ given $\theta_{*}$ (Efron and Tibshirani, 1993). We have $\sqrt{n}\left(\hat{\theta}_{1}^{*}-\hat{\theta}_{1}\right) \stackrel{d}{\longrightarrow} \sqrt{n}\left(\hat{\theta}_{1}-\theta_{1 *}\right)$ (Bickel and Freedman, 1981; Cheng and Huang 2010, Theorem $2)$, and

$$
\sqrt{n}\left(\hat{\theta}_{1}-\theta_{1 *}\right) \sim \mathbb{N}\left(0,\left[J_{1}\left(\theta_{1 *}\right)^{-1}\right]^{T} I_{1}\left(\theta_{1 *}\right) J_{1}\left(\theta_{1 *}\right)^{-1}\right),
$$

where $I_{1}\left(\theta_{1}\right)=\mathbb{E}\left\{\nabla \log f_{1}\left(X \mid \theta_{1}\right) \cdot \nabla \log f_{1}\left(X \mid \theta_{1}\right)^{T}\right\}$ (White, 1982, Theorem 3.2). Thus $\Delta A_{1}$ is a quadratic form of normal variates and is $O_{p}(1)$. If $H_{1}$ is the true model, $\Delta A_{1} \sim \frac{1}{2} \chi_{d}^{2}$ where $d$ is the number of parameters in $H_{1}$. Similarly $\Delta A_{2}=O_{p}(1)$.

We write the third term in Equation A1 as

$$
\Delta_{*}^{*} \equiv \log \frac{f_{1}\left(x^{*} \mid \hat{\theta}_{1}\right)}{f_{2}\left(x^{*} \mid \hat{\theta}_{2}\right)}=\sum_{i=1}^{n} \log \frac{f_{1}\left(x_{i}^{*} \mid \hat{\theta}_{1}\right)}{f_{2}\left(x_{i}^{*} \mid \hat{\theta}_{2}\right)} \equiv \sum_{i=1}^{n} r_{i}^{*}(x) .
$$

Define two log-likelihood ratios based on the original data $x$,

$$
\begin{aligned}
\Delta_{*} & \equiv \log \frac{f_{1}\left(x \mid \theta_{1 *}\right)}{f_{2}\left(x \mid \theta_{2 *}\right)}, \\
\Delta & \equiv \log \frac{f_{1}\left(x \mid \hat{\theta}_{1}\right)}{f_{2}\left(x \mid \hat{\theta}_{2}\right)}=\sum_{i=1}^{n} \log \frac{f_{1}\left(x_{i} \mid \hat{\theta}_{1}\right)}{f_{2}\left(x_{i} \mid \hat{\theta}_{2}\right)} \equiv \sum_{i=1}^{n} r_{i} .
\end{aligned}
$$

Note that $\Delta_{*}$ is a sum of $n$ i.i.d. terms, so that when $n \rightarrow \infty, \Delta_{*} \sim \mathbb{N}\left(0, n \sigma^{2}\right)$, with $\mathbb{E}\left(\Delta_{*}\right)=$ 
$n\left(D_{1}-D_{2}\right)=0$ (Equation 1) and $\mathbb{V}\left(\Delta_{*}\right)=n \sigma^{2}$, where

$$
\sigma^{2} \equiv \mathbb{V}_{g}\left\{\log \frac{f_{1}\left(X \mid \theta_{1 *}\right)}{f_{2}\left(X \mid \theta_{2 *}\right)}\right\}=\int g(X)\left[\log \frac{f_{1}\left(X \mid \theta_{1 *}\right)}{f_{2}\left(X \mid \theta_{2 *}\right)}\right]^{2} \mathrm{~d} X .
$$

When $n \rightarrow \infty, \bar{r}=\frac{1}{n} \sum_{i=1}^{n} r_{i} \rightarrow D_{1}-D_{2}=0$ and $s^{2}=\frac{1}{n} \sum_{i=1}^{n}\left(r_{i}-\bar{r}\right)^{2} \rightarrow \sigma^{2}$, so that $\Delta \sim \mathbb{N}\left(0, n \sigma^{2}\right)$.

Given data $x,\left\{r_{i}^{*}\right\}$ are conditionally independent, with expectation and variance

$$
\begin{aligned}
& \mathbb{E}\left(\Delta_{*}^{*} \mid x\right)=n \mathbb{E}\left\{\log \frac{f_{1}\left(x_{1}^{*} \mid \hat{\theta}_{1}\right)}{f_{2}\left(x_{1}^{*} \mid \hat{\theta}_{2}\right)} \mid x\right\} \approx n \cdot \frac{1}{n} \sum_{i=1}^{n} \log \frac{f_{1}\left(x_{i} \mid \hat{\theta}_{1}\right)}{f_{2}\left(x_{i} \mid \hat{\theta}_{2}\right)}=\sum_{i=1}^{n} r_{i}=\Delta, \\
& \mathbb{V}\left(\Delta_{*}^{*} \mid x\right)=n \mathbb{V}\left\{\log \frac{f_{1}\left(x_{1}^{*} \mid \hat{\theta}_{1}\right)}{f_{2}\left(x_{1}^{*} \mid \hat{\theta}_{2}\right)} \mid x\right\}=n \mathbb{E}\left\{\left(r_{i}^{*}-\mathbb{E}\left(r_{i}^{*}\right)\right)^{2} \mid x\right\} \approx n \sigma^{2} .
\end{aligned}
$$

Thus $\Delta_{*}^{*} \mid x \sim \mathbb{N}\left(\Delta, n \sigma^{2}\right)$. The bootstrap probability for $H_{1}$ is

$$
P_{1}=\mathbb{P}\left\{\Delta^{*}>0 \mid x\right\}=\mathbb{P}\left\{\Delta A_{1}-\Delta A_{2}+\Delta_{*}^{*}>0 \mid x\right\} \approx \mathbb{P}\left\{\Delta_{*}^{*}>0 \mid x\right\} \approx \Phi\left(\frac{\Delta}{\sqrt{n} \sigma}\right) \sim \mathbb{U}(0,1) .
$$

$P_{1}$ varies among datasets like a random number.

The case where there are no free parameters in the compared models has been discussed in the main paper. We have

$$
\Delta=\Delta_{*}=\log \frac{f_{1}(x)}{f_{2}(x)}, \quad \Delta^{*}=\Delta_{*}^{*}=\log \frac{f_{1}\left(x^{*}\right)}{f_{2}\left(x^{*}\right)},
$$

with $\Delta \sim \mathbb{N}\left(0, n \sigma^{2}\right)$ and $\Delta^{*} \sim \mathbb{N}\left(\Delta, n \sigma^{2}\right)$, as $n \rightarrow \infty$. Thus

$$
P_{1}=\mathbb{P}\left\{\Delta^{*}>0 \mid x\right\}=\Phi\left(\frac{\Delta}{\sqrt{n} \sigma}\right) \rightarrow \mathbb{U}(0,1) .
$$

The case where the two models are equally right or are equally wrong and indistinct, that is, with $f_{1}\left(X \mid \theta_{1}^{*}\right)=f_{2}\left(X \mid \theta_{2}^{*}\right)$ for almost every $X$. We have $\Delta_{*}=0$ in Equation A5, and $\Delta=O_{p}(1)$. As a result, $\Delta_{*}^{*}=O_{p}(1)$, as well as $\Delta A_{1}=O_{p}(1)$ and $\Delta A_{2}=O_{p}(1)$. From Equation $\mathrm{A} 2, \Delta A_{1}$ and $\Delta A_{2}$ have the same distribution, with $\mathbb{E}\left(\Delta A_{1}-\Delta A_{2} \mid x\right)=0$. Thus $\mathbb{E}\left(\Delta^{*} \mid x\right)=\mathbb{E}\left(\Delta_{*}^{*} \mid x\right)$ $=\Delta$. Let $F$ be the CDF of $\Delta$, which has mean 0 . Then

$$
P_{1}=\mathbb{P}\left\{\Delta^{*}>0 \mid x\right\}=1-F(-\Delta) .
$$

Thus with $n \rightarrow \infty, P_{1}$ converges to a non-degenerate distribution, which is $\mathbb{U}(0,1)$ if and only if $\Delta^{*}-\Delta$ has the same distribution as $-\Delta$.

DasGupta (2008, Chapter 29) discusses regularity conditions under which $T^{*}-T$ and $T-\mathbb{E}(T)$ have the same distribution, so that the bootstrap plugin principle can be applied, where $T$ is a statistic or function of data $x$. If those conditions are not satisfied, the standard bootstrap will fail as $T^{*}-T$ will not approximate $T-\mathbb{E}(T)$. Problem 4 is one such case, and $\Delta^{*}-\Delta$ and $\Delta$ have different distributions, and the limiting distribution of $P_{1}$ is not uniform. As indistinct models are more similar to each other than distinct models and as $P_{1} \sim \mathbb{U}(0,1)$ when the two models are distinct (and equally wrong), we conjecture that $\mathbb{V}\left(P_{1}\right) \leqslant \frac{1}{12}$, the variance of $\mathbb{U}(0,1)$.

Problems 3 and 4 are examples of equally right or equally wrong but indistinct models. Problem 3 shows the $\mathbb{U}(0,1)$ distribution, while problem 4 shows a non-uniform distribution. 
The case of $K$ models. Let the $K$ models be $H_{1}, \cdots, H_{K}$, all of which have the same K-L distance to the true model. Define

$$
\Delta_{* j k} \equiv \log \frac{f_{j}\left(x \mid \theta_{* j}\right)}{f_{k}\left(x \mid \theta_{* k}\right)}, \quad \Delta_{j k} \equiv \sum_{i=1}^{n} \log \frac{f_{j}\left(x_{i} \mid \hat{\theta}_{j}\right)}{f_{k}\left(x_{i} \mid \hat{\theta}_{k}\right)}
$$

for dataset $x$ and

$$
\Delta_{* j k}^{*} \equiv \sum_{i=1}^{n} \log \frac{f_{j}\left(x_{i}^{*} \mid \hat{\theta}_{j}\right)}{f_{k}\left(x_{i}^{*} \mid \hat{\theta}_{k}\right)}, \quad \Delta_{j k}^{*} \equiv \sum_{i=1}^{n} \log \frac{f_{j}\left(x_{i}^{*} \mid \hat{\theta}_{j}^{*}\right)}{f_{k}\left(x_{i}^{*} \mid \hat{\theta}_{k}^{*}\right)}
$$

for bootstrap dataset $x^{*}$.

First consider the case where the $K$ models are equally wrong and distinct. As in the case of two models, $\Delta_{j k}^{*}$ is dominated by $\Delta_{* j k}^{*}$ so that $\Delta_{j k}^{*} \approx \Delta_{* j k}^{*}$ while $\Delta \sim \mathbb{N}\left(0, n \sigma_{j k}^{2}\right)$ and $\Delta_{* j k}^{*} \sim$ $\mathbb{N}\left(\Delta_{j k}, n \sigma_{j k}^{2}\right)$, with $\sigma_{j k}^{2} \equiv \mathbb{V}\left\{\log \frac{f_{j}\left(X \mid \theta_{j *}\right)}{f_{k}\left(X \mid \theta_{k *}\right)}\right\}$ (see Equation A6). Given $x$, there will be a set of bootstrap probabilities $\left(P_{1}, \cdots, P_{K}\right)$. For example

$$
P_{1}=\mathbb{P}\left\{\Delta_{12}^{*}>0, \ldots, \Delta_{1 K}^{*}>0 \mid x\right\} \approx \mathbb{P}\left\{\Delta_{* 12}^{*}>0, \ldots, \Delta_{* 1 K}^{*}>0 \mid x\right\} .
$$

Let $z=\left\{z_{2}, \cdots, z_{K-1}\right\}$ and $z^{*}=\left\{z_{2}^{*}, \cdots, z_{K-1}^{*}\right\}$, where $z_{j}=\frac{\Delta_{1 j}}{\sqrt{n} \sigma_{1 j}}$ and $z_{j}^{*}=\frac{\Delta_{1 j}^{*}}{\sqrt{n} \sigma_{1 j}}$. Let

$$
\rho_{j k}=\operatorname{Cor}\left(z_{j}, z_{k}\right)=\operatorname{Cor}\left(\Delta_{1 j}, \Delta_{1 k}\right)=\frac{1}{\sigma_{1 j} \sigma_{1 k}} \operatorname{Cov}\left(\log \frac{f_{1}\left(X \mid \theta_{1 *}\right)}{f_{j}\left(X \mid \theta_{j *}\right)}, \log \frac{f_{1}\left(X \mid \theta_{1 *}\right)}{f_{k}\left(X \mid \theta_{k *}\right)}\right) .
$$

Thus $z \sim \mathbb{N}\left(0, \Sigma_{0}\right)$ and $z^{*} \mid x \sim \mathbb{N}\left(z, \Sigma_{0}\right)$, where $\Sigma_{0}$ is a $(K-1) \times(K-1)$ variance matrix with 1 on the diagonal and $\rho_{j k}$ on the off-diagonal. We have

$$
P_{1}=\mathbb{P}\left(z_{2}^{*}>0, \cdots, z_{K}^{*}>0 \mid x\right)=\Phi\left(-z_{2}, \cdots,-z_{K}\right),
$$

where $\Phi$ is the $(K-1)$-variate $\mathrm{CDF}$ of $\mathbb{N}\left(0, \Sigma_{0}\right)$. Bootstrap probabilities for the other models, $P_{2}, \cdots, P_{K}$, are given similarly.

When there is strong symmetry in the problem so that the K-L distance between any two models is the same, the variance matrix $\Sigma_{0}$ will have 1 on the diagonal and $\rho_{j k}=\frac{1}{2}$ on the off-diagonal, and further simplifications are possible. The joint distribution of bootstrap model probabilities $\left(P_{1}, \cdots, P_{K}\right)$ can be simulated as follows (see Problem 5). Sample $z=\left\{z_{2}, \cdots, z_{K}\right\} \sim$ $\mathbb{N}\left(0, \Sigma_{0}\right)$ where $\Sigma_{0}$ is $(K-1) \times(K-1)$, with 1 on the diagonal and $\frac{1}{2}$ on the off-diagonal. Let $z_{1}=-\left(z_{2}+\cdots+z_{K}\right)$. Then calculate

$$
\left[\begin{array}{c}
P_{1} \\
P_{2} \\
\vdots \\
P_{K}
\end{array}\right]=\left[\begin{array}{c}
\Phi\left(z_{2}, z_{3}, \cdots, z_{K}\right) \\
\Phi\left(-z_{2}, z_{3}-z_{2}, \cdots, z_{K}-z_{2}\right) \\
\vdots \\
\Phi\left(-z_{K}, z_{2}-z_{K}, \cdots, z_{K-1}-z_{K}\right)
\end{array}\right] .
$$$$
=0
$$
.
If the $K$ models under comparison are equally right or equally wrong and indistinct, $\Delta_{j k}^{*}$ $=O_{p}(1)$. Then the bootstrap probabilities $\left(P_{1}, \cdots, P_{K}\right)$ have a nondegenerate distribution. In the case where some of the $K$ models are equally wrong and distinct while others are indistinct, the dynamics of bootstrap support values may be complex. See table $\mathrm{S} 1$ for examples. 
(a2)

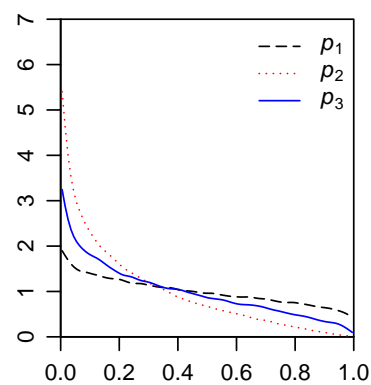

(c)

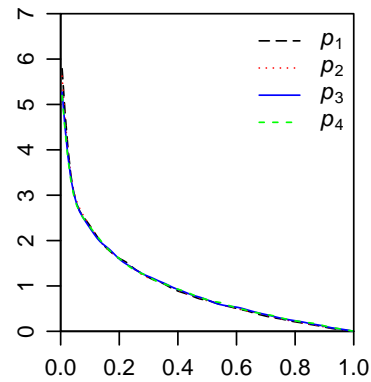

(b)

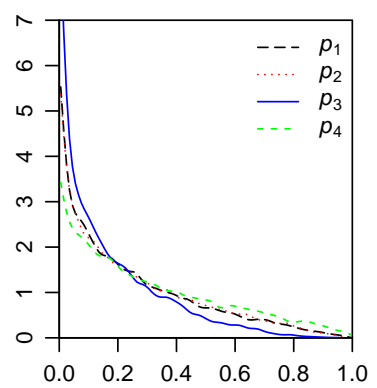

(d)

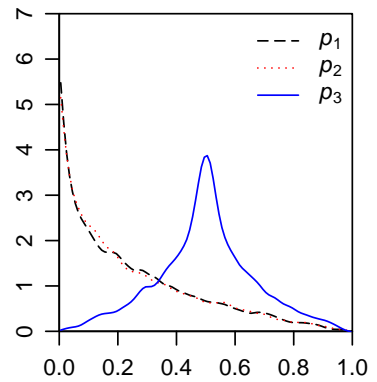

Fig. S1. The density of marginal distribution of bootstrap support $P_{i}, i=1,2,3,4$ for cases (a2),(b),(c) and (d).
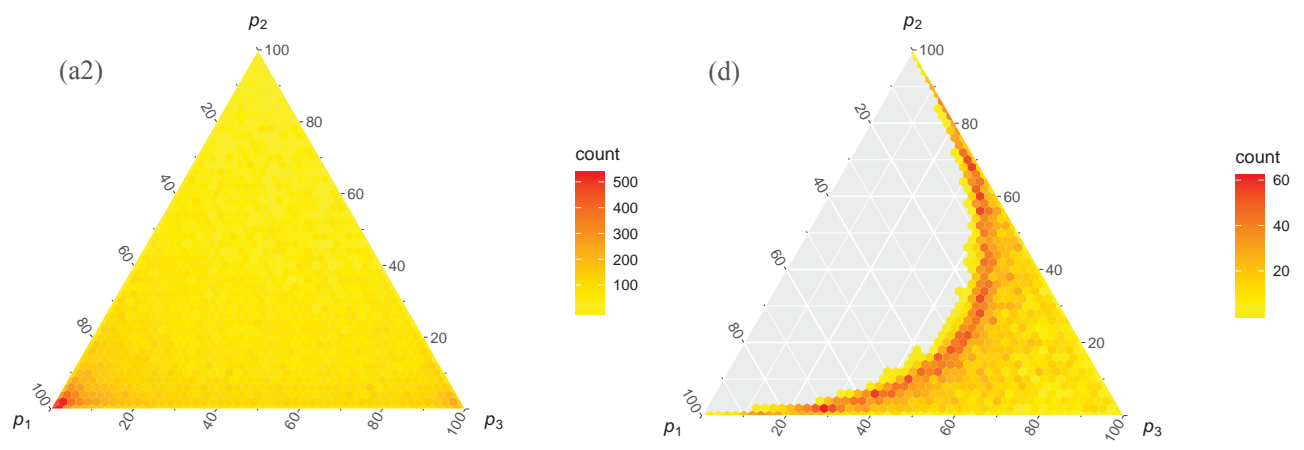

Fig. S2. Ternary distribution of bootstrap support for cases (a2) and (d).

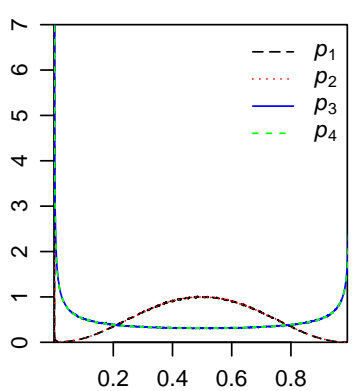

Fig. S3. The density of marginal distribution of posterior probability $P_{i}, i=1,2,3,4$ for case (c). 
SI TEXT. ANALYSIS OF PROBLEM 4: MODELS OF NORMAL MEAN AND VARIANCE

The true model is $\mathbb{N}(0,1)$ and the two compared models are $\mathbb{N}(\mu, 1)$ and $\mathbb{N}(0, v)$. In $H_{1}, \mu_{*}=0$ while in $H_{2}, v_{*}=1$, so the two models are equally right. The data $x$ may be summarized as the sample mean $\bar{x}$ and sample variance $s^{2}=\frac{1}{n} \sum_{i}\left(x_{i}-\bar{x}\right)^{2}$. The MLE of the parameter is $\hat{\mu}=\bar{x}=$ $\frac{1}{n} \sum x_{i}$ under $H_{1}$ and $\hat{v}=\overline{x^{2}}=\frac{1}{n} \sum x_{i}^{2}=\bar{x}^{2}+s^{2}$ under $H_{2}$. The log-likelihood values are

$$
\begin{aligned}
& \ell_{1}(\hat{\mu})=-\frac{1}{2} \sum\left(x_{i}-\bar{x}\right)^{2}=-\frac{1}{2} n s^{2}, \\
& \ell_{2}(\hat{v})=-\frac{n}{2} \log \left(\frac{1}{n} \sum x_{i}^{2}\right)-\frac{n}{2}=-\frac{n}{2} \log \left(s^{2}+\bar{x}^{2}\right)-\frac{n}{2}
\end{aligned}
$$

First we derive Equation 15 from first principles. Then we analyze the problem following the general theory of the Appendix. The problem has been simplified to the following. Let $z_{1}=$ $\sqrt{n} \bar{x} \sim \mathbb{N}(0,1)$ and $z_{2}=\sqrt{\frac{n}{2}}\left(s^{2}-1\right) \sim \mathbb{N}(0,1)$, and define $z_{1}^{*}$ and $z_{2}^{*}$ accordingly, with $z_{1}^{*} \mid z_{1} \sim$ $\mathbb{N}\left(z_{1}, 1\right)$ and $z_{2}^{*} \mid z_{2} \sim \mathbb{N}\left(z_{2}, 1\right)$. We seek the distribution of $P_{1}$ when $x$ varies, defined as

$$
P_{1}=\mathbb{P}\left\{\left|\bar{z}_{1}^{*}\right|>\left|\bar{z}_{2}^{*}\right| \mid z_{1}, z_{2}\right\}
$$

The CDF of the difference between two independent folded normal random variables with the same variance, $X_{1} \sim \mathbb{N}\left(\mu_{1}, \sigma^{2}\right)$ and $X_{2} \sim \mathbb{N}\left(\mu_{2}, \sigma^{2}\right)$, is

$$
\begin{aligned}
\mathbb{P}\left(\left|X_{1}\right|-\left|X_{2}\right|<t\right) & =\Phi\left(\frac{t}{\sqrt{2} \sigma}-\frac{\tilde{\mu}_{1}}{\sigma}\right)\left(1-\Phi\left(\frac{-t}{\sqrt{2} \sigma}-\frac{\tilde{\mu}_{2}}{\sigma}\right)\right) \\
& +\left(1-\Phi\left(\frac{-t}{\sqrt{2} \sigma}-\frac{\tilde{\mu}_{1}}{\sigma}\right)\right) \Phi\left(\frac{t}{\sqrt{2} \sigma}-\frac{\tilde{\mu}_{2}}{\sigma}\right)
\end{aligned}
$$

where $\left[\begin{array}{l}\tilde{\mu}_{1} \\ \tilde{\mu}_{2}\end{array}\right]=\frac{1}{\sqrt{2}}\left[\begin{array}{l}\mu_{1}-\mu_{2} \\ \mu_{1}+\mu_{2}\end{array}\right]$. Thus

$$
\begin{aligned}
P_{1} & =\Phi\left(-\frac{1}{\sqrt{2}}\left(z_{2}-z_{1}\right)\right)\left(1-\Phi\left(-\frac{1}{\sqrt{2}}\left(z_{1}+z_{2}\right)\right)\right) \\
& +\left(1-\Phi\left(-\frac{1}{\sqrt{2}}\left(z_{2}-z_{1}\right)\right)\right) \Phi\left(-\frac{1}{\sqrt{2}}\left(z_{1}+z_{2}\right)\right) \\
& \equiv Y_{1} Y_{2}+\left(1-Y_{1}\right)\left(1-Y_{2}\right),
\end{aligned}
$$

where $Y_{1}$ and $Y_{2}$ are i.i.d. from $\mathbb{U}(0,1)$. While $P_{1}$ is a function of data $x$ (Equation 14) or of $z_{1}$ and $z_{2}$ (Equation S2), it is now considered a function of $Y_{1}$ and $Y_{2}$. We use a further variable transform. Let $W_{1}=2 Y_{1}-1$ and $W_{2}=2 Y_{2}-1$, with $W_{1}$ and $W_{2}$ i.i.d. from $\mathbb{U}(-1,1)$. The density of $T=W_{1} W_{2}$ is given by the product convolution of $W_{1}$ and $W_{2}$ as

$$
f_{T}(t)=\int_{\mathbb{R}} f_{W_{1}}\left(w_{1}\right) f_{W_{2}}\left(t / w_{1}\right) \frac{1}{\left|w_{1}\right|} \mathrm{d} w_{1}=\int_{\left|w_{1}\right|<1,\left|t / w_{1}\right|<1} \frac{1}{4\left|w_{1}\right|} \mathrm{d} w_{1}=-\frac{1}{2} \log |t|,
$$

$-1<t<1$. Note that the region of integral consists of four disjoint intervals: $0<t<w_{1}<1$, $0<t<-w_{1}<1,0<-t<w_{1}<1$, and $0<-t<-w_{1}<1$. As $P_{1}=\frac{1}{2}(T+1)$, Equation 15 follows.

Next we analyze the problem by working with the log likelihood ratios as in the general theory. The log-likelihoods under the two models are

$$
\begin{aligned}
& \ell_{1}(\mu)=-\frac{1}{2} \sum\left(x_{i}-\mu\right)^{2}=-\frac{n}{2}\left(\overline{x^{2}}-2 \bar{x} \mu+\mu^{2}\right), \\
& \ell_{2}(v)=-\frac{n}{2} \log v-\frac{1}{2 v} \sum x_{i}^{2}=-\frac{n}{2}\left(\log v+\frac{1}{v} \overline{x^{2}}\right) .
\end{aligned}
$$


22

23

At the MLE $\hat{\mu}=\bar{x}$ in $H_{1}$ and $\hat{v}=\overline{x^{2}}$ in $H_{2}$, we have $\ell_{1}(\hat{\mu})=-\frac{1}{2} n\left(\overline{x^{2}}-\bar{x}^{2}\right)$ and $\ell_{2}(\hat{v})=$ $-\frac{n}{2}\left(\log \overline{x^{2}}+1\right)$, so that

$$
\begin{aligned}
\Delta & =\ell_{1}(\hat{\mu})-\ell_{2}(\hat{v})=\frac{n}{2}\left[\log \overline{x^{2}}+1-\overline{x^{2}}+\bar{x}^{2}\right] \\
& =\frac{n}{2} g(\hat{\mu}, \hat{v})=\frac{n}{2}\left[g(\hat{\mu}, \hat{v})-g\left(\mu_{*}, \nu_{*}\right)\right]
\end{aligned}
$$

Here we define a function $g(\mu, v)=\log (v)+1-v+\mu^{2}$. We have $\nabla g(\mu, v)=\left(2 \mu, \frac{1}{v}-1\right)$ and $\nabla^{2} g(\mu, v)=\left(\begin{array}{cc}2 & 0 \\ 0 & -1 / v^{2}\end{array}\right)$. Note that $g\left(\mu_{*}, v_{*}\right)=0$, and $\nabla g\left(\mu_{*}, v_{*}\right)=\left(\begin{array}{l}0 \\ 0\end{array}\right)$ and $\nabla^{2} g\left(\mu_{*}, v_{*}\right)=\left(\begin{array}{cc}2 & 0 \\ 0 & -1\end{array}\right)$. Let $\bar{Y}=\left(\bar{x}, \overline{x^{2}}\right)$ and $\mu_{Y}=\left(\mu_{*}, v_{*}\right)$, with $g\left(\mu_{Y}\right)=\Delta_{*}=0$. Then applying Taylor expansion, we get

$$
\begin{aligned}
\Delta & =\frac{n}{2}\left[g(\bar{Y})-g\left(\mu_{Y}\right)\right] \approx \frac{n}{2} \cdot \frac{1}{2}(\hat{\mu}, \hat{v}-1)\left(\begin{array}{cc}
2 & 0 \\
0 & -1
\end{array}\right)\left(\begin{array}{c}
\hat{\mu} \\
\hat{v}-1
\end{array}\right) \\
& =\frac{1}{2} n \bar{x}^{2}-\frac{1}{2} \frac{n}{2}\left(s^{2}+\bar{x}^{2}-1\right)^{2}=\frac{1}{2} z_{1}^{2}-\frac{1}{2} z_{2}^{2},
\end{aligned}
$$

where $z_{1}=\sqrt{n} \bar{x} \sim \mathbb{N}(0,1)$ and $z_{2}=\sqrt{\frac{n}{2}}\left(s^{2}+\bar{x}^{2}-1\right) \approx \sqrt{\frac{n}{2}}\left(s^{2}-1\right) \sim \mathbb{N}(0,1)$, as defined in the main text.

For the bootstrap dataset $x^{*}$, we have $\hat{\mu}^{*}=\bar{x}^{*}$ and $\hat{v}^{*}=\overline{x^{2 *}}=s^{2 *}+\bar{x}^{2 *}$. The three terms in Equation A1 can be derived as follows.

$$
\begin{aligned}
\Delta A_{1} & \equiv \log \frac{f_{1}\left(x^{*} \mid \hat{\theta}_{1}^{*}\right)}{f_{1}\left(x^{*} \mid \hat{\theta}_{1}\right)}=-\frac{1}{2} \sum\left(x_{i}^{*}-\hat{\mu}^{*}\right)^{2}+\frac{1}{2} \sum\left(x_{i}^{*}-\hat{\mu}\right)^{2} \\
& =-\frac{n}{2}\left(\overline{x^{2 *}}-\bar{x}^{2 *}-\overline{x^{2 *}}+2 \bar{x}^{*} \bar{x}-\bar{x}^{2}\right) \\
& =\frac{n}{2}\left(\bar{x}^{2 *}-2 \bar{x}^{*} \bar{x}+\bar{x}^{2}\right)=\frac{n}{2}\left(\bar{x}^{*}-\bar{x}\right)^{2}=\frac{1}{2}\left(z_{1}^{*}-z_{1}\right)^{2},
\end{aligned}
$$

with $z_{1}^{*}$ and $z_{2}^{*}$ defined above. Similarly

$$
\begin{aligned}
\Delta A_{2} & \equiv \log \frac{f_{2}\left(x^{*} \mid \hat{\theta}_{2}^{*}\right)}{f_{2}\left(x^{*} \mid \hat{\theta}_{2}\right)}=-\frac{n}{2}\left(\log \hat{v}^{*}+\frac{\overline{x^{2 *}}}{\hat{v}^{*}}\right)+\frac{n}{2}\left(\log \hat{v}+\frac{\overline{x^{2 *}}}{\hat{v}}\right) \\
& =-\frac{n}{2}\left[\left(\log \hat{v}^{*}-\frac{\hat{v}^{*}}{\hat{v}}\right)-(\log \hat{v}-1)\right] \\
& \approx-\frac{n}{2} \cdot 0 \cdot\left(\hat{v}^{*}-\hat{v}\right)-\frac{n}{2} \cdot \frac{1}{2}\left(-\frac{1}{\hat{v}^{2}}\right)\left(\hat{v}^{*}-\hat{v}\right)^{2} \approx \frac{n}{4}\left(\hat{v}^{*}-\hat{v}\right)^{2}=\frac{1}{2}\left(z_{2}^{*}-z_{2}\right)^{2} .
\end{aligned}
$$

Here in the Taylor expansion $\hat{v}$ is constant and $\hat{v}^{*}$ is a random variable given $x$.

$$
\begin{aligned}
\Delta_{*}^{*} & \equiv \log \frac{f_{1}\left(x^{*} \mid \hat{\theta}_{1}\right)}{f_{2}\left(x^{*} \mid \hat{\theta}_{2}\right)}=-\frac{1}{2} \sum\left(x_{i}^{*}-\hat{\mu}\right)^{2}+\frac{n}{2}\left(\log \hat{v}+\frac{\overline{x^{2 *}}}{\hat{v}}\right)=\frac{n}{2}\left(-\hat{v}^{*}+2 \bar{x}^{*} \bar{x}-\bar{x}^{2}+\log \hat{v}+\frac{\hat{v}^{*}}{\hat{v}}\right) \\
& \approx \frac{n}{2}\left(2 \bar{x}^{*} \bar{x}-2 \bar{x}^{2}\right)+\frac{n}{2} \bar{x}^{2}+\frac{n}{2}\left[\left(\hat{v}^{*}\right.\right. \\
\hat{v} & \left.\left.\hat{v}^{*}\right)-(1-\hat{v})\right]+\frac{n}{2}(\log \hat{v}+1-\hat{v}) \\
& \approx n \bar{x}\left(\bar{x}^{*}-\bar{x}\right)+\frac{n}{2}\left(\frac{1}{\hat{v}}-1\right)\left(\hat{v}^{*}-\hat{v}\right)+\frac{n}{2}\left(\bar{x}^{2}+\log \hat{v}+1-\hat{v}\right) \\
& =n \bar{x}\left(\bar{x}^{*}-\bar{x}\right)-\frac{n}{2} \frac{1}{\hat{v}}(\hat{v}-1)\left(\hat{v}^{*}-\hat{v}\right)+\Delta \approx z_{1}\left(z_{1}^{*}-z_{1}\right)-z_{2}\left(z_{2}^{*}-z_{2}\right)+\Delta .
\end{aligned}
$$

Thus

$$
\begin{aligned}
\Delta^{*} & =\Delta A_{1}-\Delta A_{2}+\Delta_{*}^{*} \\
& \approx \frac{1}{2}\left(z_{1}^{*}-z_{1}\right)^{2}-\frac{1}{2}\left(z_{2}^{*}-z_{2}\right)^{2}+z_{1}\left(z_{1}^{*}-z_{1}\right)-z_{2}\left(z_{2}^{*}-z_{2}\right)+\frac{1}{2}\left(z_{1}^{2}-z_{2}^{2}\right) \\
& =\frac{1}{2}\left(z_{1}^{* 2}-z_{2}^{* 2}\right) .
\end{aligned}
$$


${ }_{34}$ This can also be obtained by a Taylor expansion of the function $g()$,

$$
\begin{aligned}
\Delta^{*}-\Delta & =\frac{n}{2}\left[g\left(\bar{Y}^{*}\right)-g(\bar{Y})\right]=\frac{n}{2}\left[g\left(\hat{\mu}^{*}, \hat{v}^{*}\right)-g(\hat{\mu}, \hat{v})\right] \\
& \approx \frac{n}{2}\left(2 \hat{\mu}, \frac{1}{\hat{v}}-1\right)\left(\begin{array}{c}
\hat{\mu}^{*}-\hat{\mu} \\
\hat{v}^{*}-\hat{v}
\end{array}\right)+\frac{n}{4}\left(\hat{\mu}^{*}-\hat{\mu}, \hat{v}^{*}-\hat{v}\right) \nabla^{2} g(\hat{\mu}, \hat{v})\left(\begin{array}{c}
\hat{\mu}^{*}-\hat{\mu} \\
\hat{v}^{*}-\hat{v}
\end{array}\right) \\
& =z_{1}\left(z_{1}^{*}-z_{1}\right)-z_{2}\left(z_{2}^{*}-z_{2}\right)+\frac{1}{2}\left(z_{1}^{*}-z_{1}\right)^{2}-\frac{1}{2}\left(z_{2}^{*}-z_{2}\right)^{2} .
\end{aligned}
$$

Thus Equation $\mathrm{S} 12$ gives $P_{1}=\mathbb{P}\left\{\Delta^{*}>0 \mid x\right\}=\mathbb{P}\left\{z_{1}^{* 2}-z_{2}^{* 2} \mid x\right\}$, as in Equation S2, and Equation 15 follows.

Note that

$$
P_{1}=\mathbb{P}\left\{\Delta^{*}>0 \mid x\right\}=\mathbb{P}\left\{\Delta^{*}-\Delta>-\Delta \mid x\right\}=1-F_{\Delta^{*}-\Delta}(-\Delta),
$$

where $F(\cdot)$ is the CDF of $\Delta^{*}-\Delta$. We note that being the difference of two $\chi_{d}^{2}$ variates, $\Delta$ has the same distribution as $-\Delta$, which may and may not be the same distribution as that of $\Delta^{*}-\Delta$.

When $\Delta^{*}-\Delta$ and $-\Delta$ have the same distribution, $P_{1} \sim \mathbb{U}(0,1)$. Otherwise if $\Delta^{*}-\Delta$ and $-\Delta$ have different distributions, $P_{1}$ does not have a uniform distribution. This follows from the fact that if $F$ is a smooth monotonic increasing function $\mathbb{R} \rightarrow(0,1)$, and $Y$ is a random variable over $\mathbb{R}$, then $F(Y) \sim \mathbb{U}(0,1)$ implies that $F$ is the CDF of $Y$, because the CDF of $Y$ is $F_{Y}(y)=\mathbb{P}(Y<y)=\mathbb{P}(F(Y)<F(y))=F(y)$.

According to DasGupta (2008, p. 475), if $T=\sqrt{n}\left(g(\bar{Y})-g\left(\theta_{*}\right)\right)$ and $\nabla g\left(\theta_{*}\right)=0$, then $T$ and $T^{*}$ have different distributions and bootstrap fails to estimate the CDF of $T$ consistently. In problem $4, \nabla g\left(\mu_{*}, \nu_{*}\right)=0$, so that $\Delta^{*}-\Delta$ has a different distribution from $\Delta-\Delta_{*}$, and $P_{1}$ is not distributed as $\mathbb{U}(0,1)$. In problems 1 and $2, \Delta^{*}-\Delta$ and $\Delta-\Delta_{*}$ have the same distribution, so that $P_{1} \sim \mathbb{U}(0,1)$ 


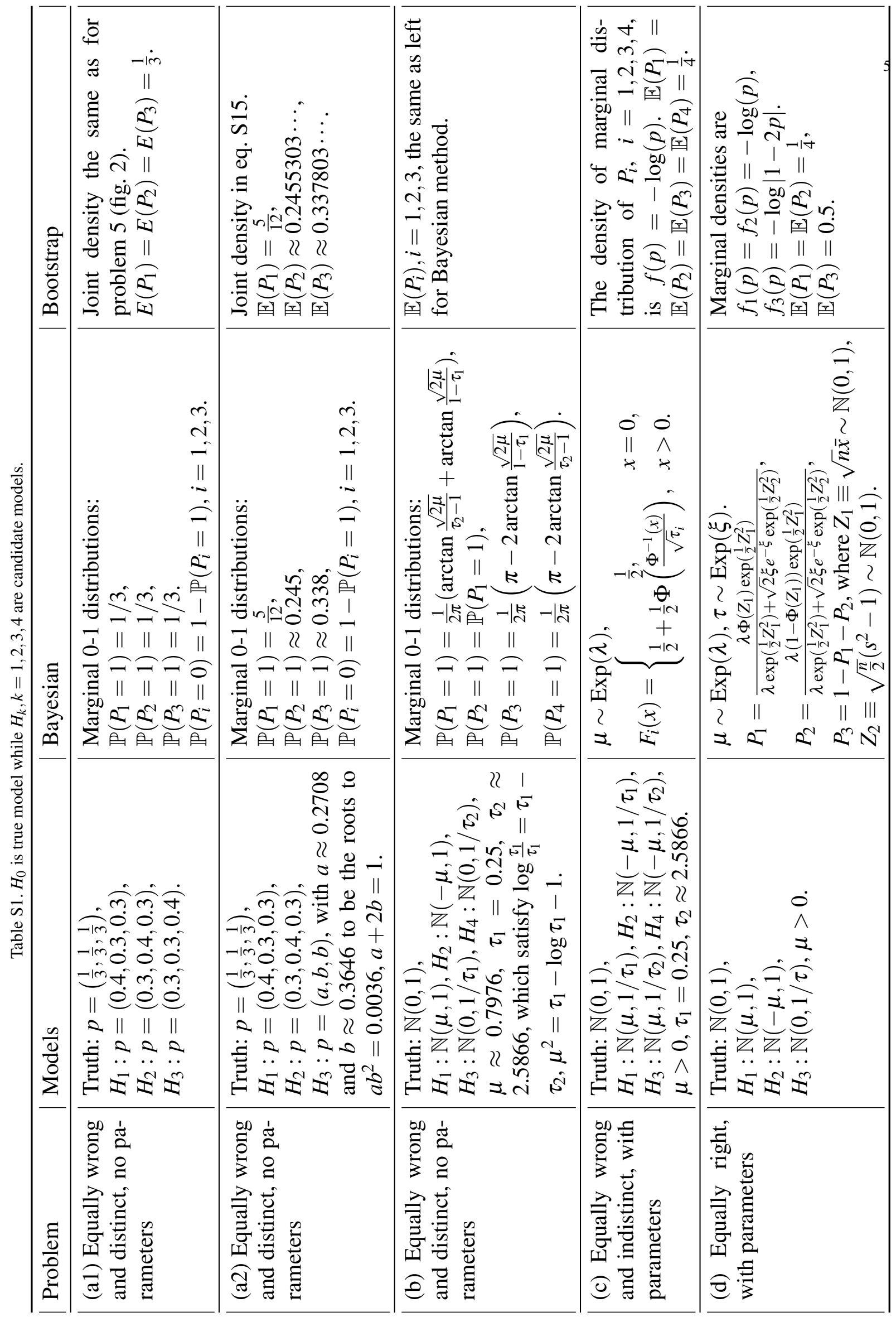


${ }_{50} \quad$ Note. - For case a2, let $h=\frac{\log (0.3)-\log (b)}{\log (0.4)-\log (b)} \approx-2.102287$. The correlation matrix for $\Delta_{12}, \Delta_{13}, \Delta_{23}$ 51 is

$$
\left(\begin{array}{ccc}
1 & \frac{\sqrt{3}}{2} & -\frac{h+2}{2 \sqrt{h^{2}+h+1}} \\
& 1 & -\sqrt{\frac{3}{4}} \cdot \frac{h+1}{\sqrt{h^{2}+h+1}}
\end{array}\right)
$$

${ }_{52}$ The expectations are

53

54

55

$$
\begin{aligned}
& \mathbb{E}\left(P_{1}\right)=\frac{1}{4}+\frac{1}{2 \pi} \arcsin \rho\left(\frac{\Delta_{12}}{\sqrt{n} \sigma_{12}}, \frac{\Delta_{13}}{\sqrt{n} \sigma_{13}}\right)=\frac{5}{12}, \\
& \mathbb{E}\left(P_{2}\right)=\frac{1}{4}+\frac{1}{2 \pi} \arcsin \rho\left(-\frac{\Delta_{12}}{\sqrt{n} \sigma_{12}}, \frac{\Delta_{23}}{\sqrt{n} \sigma_{23}}\right)=0.2455303 \cdots, \\
& \mathbb{E}\left(P_{3}\right)=\frac{1}{4}+\frac{1}{2 \pi} \arcsin \rho\left(\frac{\Delta_{13}}{\sqrt{n} \sigma_{13}}, \frac{\Delta_{23}}{\sqrt{n} \sigma_{23}}\right)=0.337803 \cdots .
\end{aligned}
$$

